\title{
Localization of Cohomological Induction
}

\author{
by
}

Yoshiki Oshima

\begin{abstract}
We give a geometric realization of cohomologically induced $(\mathfrak{g}, K)$-modules. Let $(\mathfrak{h}, L)$ be a subpair of $(\mathfrak{g}, K)$. The cohomological induction is an algebraic construction of $(\mathfrak{g}, K)$ modules from an $(\mathfrak{h}, L)$-module $V$. For a real semisimple Lie group, the duality theorem by Hecht, Miličić, Schmid, and Wolf relates $(\mathfrak{g}, K)$-modules cohomologically induced from a Borel subalgebra to $\mathcal{D}$-modules on the flag variety of $\mathfrak{g}$. In this article we extend the theorem to more general pairs $(\mathfrak{g}, K)$ and $(\mathfrak{h}, L)$. We consider the tensor product of a $\mathcal{D}$-module and a certain module associated with $V$, and prove that its sheaf cohomology groups are isomorphic to cohomologically induced modules.
\end{abstract}

2010 Mathematics Subject Classification: Primary 22E47; Secondary 14F05, 20G20.

Keywords: Harish-Chandra module, reductive group, algebraic group, D-module, cohomological induction, Zuckerman functor.

\section{$\S 1$. Introduction}

The aim of this article is to realize cohomologically induced modules as sheaf cohomology groups of certain sheaves on homogeneous spaces.

Cohomological induction is defined as a functor between the categories of $(\mathfrak{g}, K)$-modules. Let $(\mathfrak{g}, K)$ be a pair (Definition 2.1) and let $\mathcal{C}(\mathfrak{g}, K)$ be the category of $(\mathfrak{g}, K)$-modules. Suppose that $(\mathfrak{h}, L)$ is a subpair of $(\mathfrak{g}, K)$ and that $K$ and $L$ are reductive. Following the book by Knapp and Vogan [KV95], we define the functors $P_{\mathfrak{h}, L}^{\mathfrak{g}, K}$ and $I_{\mathfrak{h}, L}^{\mathfrak{g}, K}: \mathcal{C}(\mathfrak{h}, L) \rightarrow \mathcal{C}(\mathfrak{g}, K)$ as $V \mapsto R(\mathfrak{g}, K) \otimes_{R(\mathfrak{h}, L)} V$ and $V \mapsto\left(\operatorname{Hom}_{R(\mathfrak{h}, L)}(R(\mathfrak{g}, K), V)\right)_{K}$, respectively. See Section 2 for the definition of the Hecke algebra $R(\mathfrak{g}, K)$. When $\mathfrak{g}=\mathfrak{h}$, the functor $I_{\mathfrak{h}, L}^{\mathfrak{g}, K}=I_{\mathfrak{g}, L}^{\mathfrak{g}, K}$ is called the Zuckerman functor. Let $V$ be an $(\mathfrak{h}, L)$-module. We define the cohomologically induced module as the $(\mathfrak{g}, K)$-module $\left(P_{\mathfrak{h}, L}^{\mathfrak{g}, K}\right)_{j}(V)$ for $j \in \mathbb{N}$, where $\left(P_{\mathfrak{h}, L}^{\mathfrak{g}, K}\right)_{j}$ is the

Communicated by H. Nakajima. Received July 27, 2012. Revised December 2, 2012.

Y. Oshima: Kavli IPMU (WPI), The University of Tokyo,

5-1-5 Kashiwanoha, Kashiwa, 277-8583 Chiba, Japan;

e-mail: yoshiki.oshima@ipmu.jp 
$j$-th left derived functor of $P_{\mathfrak{h}, L}^{\mathfrak{g}, K}$. Similarly, we define $\left(I_{\mathfrak{h}, L}^{\mathfrak{g}, K}\right)^{j}(V)$, where $\left(I_{\mathfrak{h}, L}^{\mathfrak{g}, K}\right)^{j}$ is the $j$-th right derived functor of $I_{\mathfrak{h}, L}^{\mathfrak{g}, K}$.

This construction produces a large family of representations of real reductive Lie groups. Let $G_{\mathbb{R}}$ be a real reductive Lie group with a Cartan involution $\theta$ so that the group of fixed points $K_{\mathbb{R}}:=\left(G_{\mathbb{R}}\right)^{\theta}$ is a maximal compact subgroup. Let $\mathfrak{g}$ be the complexified Lie algebra of $G_{\mathbb{R}}$ and $K$ the complexification of $K_{\mathbb{R}}$. We give examples of cohomologically induced $(\mathfrak{g}, K)$-modules below. In the following three examples we suppose that $\mathfrak{h}$ is a parabolic subalgebra of $\mathfrak{g}$, and $L$ is a maximal reductive subgroup of the normalizer $N_{K}(\mathfrak{h})$. We also suppose that $V$ is a onedimensional $(\mathfrak{h}, L)$-module.

- We assume the rank condition $\operatorname{rank} \mathfrak{g}=\operatorname{rank} K$ and that $\mathfrak{h}$ is a $\theta$-stable Borel subalgebra. Then under a certain positivity condition on $V,\left(P_{\mathfrak{h}, L}^{\mathfrak{g}, K}\right)_{s}(V)$ (or $\left.\left(I_{\mathfrak{h}, L}^{\mathfrak{g}, K}\right)^{s}(V)\right)$ is the underlying $(\mathfrak{g}, K)$-module of a discrete series representation of $G_{\mathbb{R}}$. Here $s=\frac{1}{2} \operatorname{dim} K / L$.

- Suppose that $\mathfrak{h}$ is a $\theta$-stable parabolic subalgebra. Then the $(\mathfrak{g}, K)$-module $\left(P_{\mathfrak{h}, L}^{\mathfrak{g}, K}\right)_{s}(V)\left(\operatorname{or}\left(I_{\mathfrak{h}, L}^{\mathfrak{g}, K}\right)^{s}(V)\right)$ is called Zuckerman's derived functor module $A_{\mathfrak{h}}(\lambda)$. Here $s=\frac{1}{2} \operatorname{dim} K / L$.

- Let $P_{\mathbb{R}}$ be a parabolic subgroup of $G_{\mathbb{R}}$ and suppose that $\mathfrak{h}$ is its complexified Lie algebra. Then $\left(P_{\mathfrak{h}, L}^{\mathfrak{g}, K}\right)_{0}(V)$ (or $\left.\left(I_{\mathfrak{h}, L}^{\mathfrak{g}, K}\right)^{0}(V)\right)$ is the underlying $(\mathfrak{g}, K)$-module of a degenerate principal series representation realized on the real flag variety $G_{\mathbb{R}} / P_{\mathbb{R}}$

The localization theory by Beilinson-Bernstein [BB81] provides another important construction of $(\mathfrak{g}, K)$-modules. It gives a realization of $(\mathfrak{g}, K)$-modules as $K$-equivariant twisted $\mathcal{D}$-modules on the full flag variety $X$ of $\mathfrak{g}$.

These two constructions are related by a result of Hecht-Miličić-SchmidWolf [HMSW87]. We now recall their theorem. Let $G_{\mathbb{R}}$ be a connected real reductive Lie group and let $(\mathfrak{g}, K)$ be the pair defined in the above way. Suppose that $\mathfrak{h}=\mathfrak{b}$ is a Borel subalgebra of $\mathfrak{g}$ and $L$ is a maximal reductive subgroup of the normalizer $N_{K}(\mathfrak{b})$. Let $X$ be the full flag variety of $\mathfrak{g}, Y$ the $K$-orbit through $\mathfrak{b} \in X$, and $i: Y \rightarrow X$ the inclusion map. Suppose that $V$ is a $(\mathfrak{b}, L)$-module and $\mathfrak{b}$ acts as scalars given by $\lambda \in \mathfrak{b}^{*}:=\operatorname{Hom}_{\mathbb{C}}(\mathfrak{b}, \mathbb{C})$. Write $\mathcal{V}_{Y}$ for the corresponding locally free $\mathcal{O}_{Y}$-module on $Y$ and view it as a twisted $\mathcal{D}$-module. Let $\mathcal{D}_{X, \lambda}$ be the ring of twisted differential operators on $X$ corresponding to $\lambda$ and define the $\mathcal{D}_{X, \lambda}$-module direct image $i_{+} \mathcal{V}_{Y}$. Then the following is called the duality theorem:

Theorem 1.1 ([HMSW87]). There is an isomorphism of $(\mathfrak{g}, K)$-modules

$$
\mathrm{H}^{s}\left(X, i_{+} \mathcal{V}_{Y}\right)^{*} \simeq\left(I_{\mathfrak{b}, L}^{\mathfrak{g}, K}\right)^{u-s}\left(V^{*} \otimes \bigwedge^{\mathrm{top}}(\mathfrak{g} / \mathfrak{b})^{*}\right)
$$


for $s \in \mathbb{N}$ and $u=\operatorname{dim} K / L-\operatorname{dim} Y$. Here the left side is the $K$-finite dual of the $(\mathfrak{g}, K)$-module $\mathrm{H}^{s}\left(X, i_{+} \mathcal{V}_{Y}\right)$.

The proof in [HMSW87] is by describing the cohomology groups of both sides by using standard resolutions and giving an isomorphism between the two complexes. We note that by using the dual isomorphism ([KV95, Theorem 3.1]) $\left(P_{\mathfrak{h}, L}^{\mathfrak{g}, K}\right)_{j}(V)^{*} \simeq\left(I_{\mathfrak{h}, L}^{\mathfrak{g}, K}\right)^{j}\left(V^{*}\right)$, Theorem 1.1 can be deduced from

$$
\mathrm{H}^{s}\left(X, i_{+} \mathcal{V}_{Y}\right) \simeq\left(P_{\mathfrak{b}, L}^{\mathfrak{g}, K}\right)_{u-s}\left(V \otimes \bigwedge^{\mathrm{top}}(\mathfrak{g} / \mathfrak{b})\right) .
$$

The relation between cohomological induction and localization has been studied further (see [Bie90], [Cha93], [Kit10], [MP98], [Sch91]). Miličić-Pandžić [MP98] gave a more conceptual proof of Theorem 1.1 by using equivariant derived categories. In [Cha93] and [Kit10], Theorem 1.1 was extended to the case of partial flag varieties.

In this article we will realize geometrically the cohomologically induced modules in the following setting. Let $i: K \rightarrow G$ be a homomorphism between complex linear algebraic groups. Suppose that $K$ is reductive and the kernel of $i$ is finite so that the pair $(\mathfrak{g}, K)$ is defined. Let $H$ be a closed subgroup of $G$. Put $M:=i^{-1}(H)$ and take a Levi decomposition $M=L \ltimes U$. We write $i: Y=K / M \rightarrow G / H=X$ for the natural immersion. Let $V$ be an $(\mathfrak{h}, M)$-module. We view $V$ as an $(\mathfrak{h}, L)$ module by restriction and define the cohomologically induced module $\left(P_{\mathfrak{h}, L}^{\mathfrak{g}, K}\right)_{j}(V)$. In this generality, we can no longer realize it as a (twisted) $\mathcal{D}$-module on $X=G / H$. Instead we use the tensor product of an $i^{-1} \mathcal{D}_{X}$-module and an $i^{-1} \mathcal{O}_{X}$-module associated with $V$ which is equipped with a $(\mathfrak{g}, K)$-action (see Definition 3.3). We now state the main theorem of this article.

Main Theorem (Theorem 4.1). Suppose that $\mathcal{V}$ is an $i^{-1} \widetilde{\mathfrak{g}}_{X}$-module associated with $V$ (see Definition 3.3). Then we have an isomorphism of $(\mathfrak{g}, K)$-modules

$$
\mathrm{H}^{s}\left(Y, i^{-1} i_{+} \mathcal{L} \otimes_{i^{-1} \mathcal{O}_{X}} \mathcal{V}\right) \simeq\left(P_{\mathfrak{h}, L}^{\mathfrak{g}, K}\right)_{u-s}\left(V \otimes \bigwedge^{\text {top }}(\mathfrak{g} / \mathfrak{h})\right)
$$

for $s \in \mathbb{N}$ and $u=\operatorname{dim} U$.

Here $\mathcal{L}$ is the invertible sheaf on $Y$ defined at the beginning of Section 4 and the direct image $i_{+} \mathcal{L}$ in the categories of $\mathcal{D}$-modules is defined as

$$
i_{*}\left(\left(\mathcal{L} \otimes_{\mathcal{O}_{Y}} \Omega_{Y}\right) \otimes_{\mathcal{D}_{Y}} i^{*} \mathcal{D}_{X}\right) \otimes_{\mathcal{O}_{X}} \Omega_{X}^{\vee} .
$$

Hence its inverse image $i^{-1} i_{+} \mathcal{L}$ as a sheaf of abelian groups is given by

$$
\left(\mathcal{L} \otimes_{\mathcal{O}_{Y}} \Omega_{Y}\right) \otimes_{\mathcal{D}_{Y}} i^{*} \mathcal{D}_{X} \otimes_{i^{-1} \mathcal{O}_{X}} i^{-1} \Omega_{X}^{\vee}
$$


We note that if $V$ comes from an algebraic $H$-module, then we can take $\mathcal{V}$ to be $i^{-1} \mathcal{V}_{X}$, where $\mathcal{V}_{X}$ is a $G$-equivariant locally free $\mathcal{O}_{X}$-module with typical fiber $V$ (Example 3.5).

The work in this article was motivated by the study of branching laws of representations. In [Osh11] a special case of Theorem 4.1 was proved and it was used to get an estimate of the restriction of $A_{\mathfrak{q}}(\lambda)$ to reductive subalgebras.

This article is organized as follows. In Section 2 we recall the definition of cohomological induction following [KV95]. In Section 3 we give the definition of an $i^{-1} \mathcal{O}$-module associated with an $(\mathfrak{h}, M)$-module. We state and prove the main theorem (Theorem 4.1) in Section 4. Our proof basically follows the proof of the duality theorem in [HMSW87]. Section 5 is devoted to the construction of the $i^{-1} \mathcal{O}$-module associated with an $(\mathfrak{h}, M)$-module, which can be used for the geometric realization of cohomologically induced modules. In Section 6, we see that the module $i^{-1} i_{+} \mathcal{L} \otimes \mathcal{V}$ can be viewed as a twisted $\mathcal{D}$-module if $\mathfrak{h}$ acts as scalars on $V$. Therefore, Theorem 4.1 becomes the isomorphism (1.1) and hence Theorem 1.1 in the particular setting.

\section{§2. Cohomological induction}

In this section we recall the definition of cohomological induction following [KV95].

Let $K$ be a complex reductive algebraic group and let $K_{\mathbb{R}}$ be a compact real form. Since any locally finite action of $K_{\mathbb{R}}$ uniquely extends to an algebraic action of $K$, the locally finite $K_{\mathbb{R}}$-modules are identified with the algebraic $K$-modules. Define the Hecke algebra $R\left(K_{\mathbb{R}}\right)$ as the space of $K_{\mathbb{R}}$-finite distributions on $K_{\mathbb{R}}$. For $S \in R\left(K_{\mathbb{R}}\right)$, the pairing with a smooth function $f$ on $K_{\mathbb{R}}$ is written as

$$
\int_{K_{\mathbb{R}}} f(k) d S(k)
$$

The product of $S, T \in R\left(K_{\mathbb{R}}\right)$ is given by

$$
S * T: f \mapsto \int_{K_{\mathbb{R}} \times K_{\mathbb{R}}} f\left(k k^{\prime}\right) d S(k) d T\left(k^{\prime}\right) .
$$

The associative algebra $R\left(K_{\mathbb{R}}\right)$ does not have the identity, but has an approximate identity (see [KV95, Chapter I]). The locally finite $K_{\mathbb{R}}$-modules are identified with the approximately unital left $R\left(K_{\mathbb{R}}\right)$-modules. The action map $R\left(K_{\mathbb{R}}\right) \times V \rightarrow V$ is given by

$$
(S, v) \mapsto \int_{K_{\mathbb{R}}} k v d S(k)
$$


for a locally finite $K_{\mathbb{R}}$-module $V$. Here, $k v$ is regarded as a smooth function on $K_{\mathbb{R}}$ that takes values in $V$. We have a natural isomorphism of $\mathbb{C}$-algebras

$$
R\left(K_{\mathbb{R}}\right) \simeq \bigoplus_{\tau \in \widehat{K}} \operatorname{End}_{\mathbb{C}}\left(V_{\tau}\right),
$$

where $\widehat{K}$ is the set of equivalence classes of irreducible $K$-modules, and $V_{\tau}$ is a representation space of $\tau \in \widehat{K}$. Hence $R\left(K_{\mathbb{R}}\right)$ depends only on the complexification $K$ up to natural isomorphisms, so in what follows, we also denote $R\left(K_{\mathbb{R}}\right)$ by $R(K)$.

Definition 2.1. Let $\mathfrak{g}$ be a Lie algebra and $K$ a complex linear algebraic group such that the Lie algebra $\mathfrak{k}$ of $K$ is a subalgebra of $\mathfrak{g}$. Suppose that a homomorphism $\phi: K \rightarrow \operatorname{Aut}(\mathfrak{g})$ of algebraic groups is given, where $\operatorname{Aut}(\mathfrak{g})$ is the automorphism group of $\mathfrak{g}$. We say $(\mathfrak{g}, K)$ is a pair if

- $\left.\phi(\cdot)\right|_{\mathfrak{k}}$ is equal to the adjoint action $\operatorname{Ad}_{\mathfrak{k}}(K)$ of $K$, and

- the differential of $\phi$ is equal to the adjoint action $\operatorname{ad}_{\mathfrak{g}}(\mathfrak{k})$.

Let $i: K \rightarrow G$ be a homomorphism of complex linear algebraic groups with finite kernel and let $\mathfrak{g}$ be the Lie algebra of $G$. Then $(\mathfrak{g}, K)$ with the homomorphism $\phi:=\operatorname{Ad} \circ i$ is a pair in the above sense.

Definition 2.2. Let $(\mathfrak{g}, K)$ be a pair. Let $V$ be a complex vector space with a Lie algebra action of $\mathfrak{g}$ and an algebraic action of $K$. We say that $V$ is a $(\mathfrak{g}, K)$-module if

- the differential of the action of $K$ coincides with the restriction of the action of $\mathfrak{g}$ to $\mathfrak{k}$, and

- $(\phi(k) \xi) v=k\left(\xi\left(k^{-1}(v)\right)\right)$ for $k \in K, \xi \in \mathfrak{g}$, and $v \in V$.

For a pair $(\mathfrak{g}, K)$, we denote by $\mathcal{C}(\mathfrak{g}, K)$ the category of $(\mathfrak{g}, K)$-modules. Suppose moreover that $K$ is reductive. We extend the representation $\phi: K \rightarrow \operatorname{Aut}(\mathfrak{g})$ to a representation $\phi: K \rightarrow \operatorname{Aut}(U(\mathfrak{g}))$ on the universal enveloping algebra. We define the Hecke algebra $R(\mathfrak{g}, K)$ as

$$
R(\mathfrak{g}, K):=R(K) \otimes_{U(\mathfrak{k})} U(\mathfrak{g}) .
$$

The product is given by

$$
(S \otimes \xi) \cdot(T \otimes \eta)=\sum_{i}\left(S *\left(\left\langle\xi_{i}^{*}, \phi(\cdot)^{-1} \xi\right\rangle T\right) \otimes \xi_{i} \eta\right)
$$

for $S, T \in R(K)$ and $\xi, \eta \in U(\mathfrak{g})$. Here $\xi_{i}$ is a basis of the linear span of $\phi(K) \xi$, and $\xi_{i}^{*}$ is its dual basis. We regard $\left\langle\xi_{i}^{*}, \phi(\cdot)^{-1} \xi\right\rangle$ as a function on $K_{\mathbb{R}}$. As in the 
group case, the $(\mathfrak{g}, K)$-modules are identified with the approximately unital left $R(\mathfrak{g}, K)$-modules. The action map $R(\mathfrak{g}, K) \times V \rightarrow V$ is given by

$$
(S \otimes \xi, v) \mapsto \int_{K_{\mathbb{R}}} k(\xi v) d S(k)
$$

for a $(\mathfrak{g}, K)$-module $V$.

Let $(\mathfrak{g}, K)$ and $(\mathfrak{h}, L)$ be pairs in the sense of Definition 2.1. Suppose that $K$ and $L$ are reductive. Let $i:(\mathfrak{h}, L) \rightarrow(\mathfrak{g}, K)$ be a map between pairs, namely, a Lie algebra homomorphism $i_{\text {alg }}: \mathfrak{h} \rightarrow \mathfrak{g}$ and an algebraic group homomorphism $i_{\mathrm{gp}}: L \rightarrow K$ satisfy the following two assumptions:

- The restriction of $i_{\text {alg }}$ to the Lie algebra $\mathfrak{l}$ of $L$ is equal to the differential of $i_{\mathrm{gp}}$. - $\phi_{K}\left(i_{\mathrm{gp}}(l)\right) \circ i_{\mathrm{alg}}=i_{\mathrm{alg}} \circ \phi_{L}(l)$ for $l \in L$, where $\phi_{K}$ denotes $\phi$ for $(\mathfrak{g}, K)$ in Definition 2.1 and $\phi_{L}$ denotes $\phi$ for $(\mathfrak{h}, L)$.

We define the functors $P_{\mathfrak{h}, L}^{\mathfrak{g}, K}, I_{\mathfrak{h}, L}^{\mathfrak{g}, K}: \mathcal{C}(\mathfrak{h}, L) \rightarrow \mathcal{C}(\mathfrak{g}, K)$ by

$$
\begin{aligned}
& P_{\mathfrak{h}, L}^{\mathfrak{g}, K}: V \mapsto R(\mathfrak{g}, K) \otimes_{R(\mathfrak{h}, L)} V, \\
& I_{\mathfrak{h}, L}^{\mathfrak{g}, K}: V \mapsto\left(\operatorname{Hom}_{R(\mathfrak{h}, L)}(R(\mathfrak{g}, K), V)\right)_{K},
\end{aligned}
$$

where $(\cdot)_{K}$ is the subspace of $K$-finite vectors. Then $P_{\mathfrak{h}, L}^{\mathfrak{g}, K}$ is right exact and $I_{\mathfrak{h}, L}^{\mathfrak{g}, K}$ is left exact. Write $\left(P_{\mathfrak{h}, L}^{\mathfrak{g}, K}\right)_{j}$ for the $j$-th left derived functor of $P_{\mathfrak{h}, L}^{\mathfrak{g}, K}$ and write $\left(I_{\mathfrak{h}, L}^{\mathfrak{g}, K}\right)^{j}$ for the $j$-th right derived functor of $I_{\mathfrak{h}, L}^{\mathfrak{g}, K}$. We can see that $I_{\mathfrak{h}, L}^{\mathfrak{g}, K}$ is the right adjoint functor of the forgetful functor

$$
\operatorname{For}_{\mathfrak{g}, K}^{\mathfrak{h}, L}: \mathcal{C}(\mathfrak{g}, K) \rightarrow \mathcal{C}(\mathfrak{h}, L), \quad V \mapsto R(\mathfrak{g}, K) \otimes_{R(\mathfrak{g}, K)} V \simeq V
$$

and $P_{\mathfrak{h}, L}^{\mathfrak{g}, K}$ is the left adjoint functor of the functor

$$
\operatorname{For}_{\mathfrak{g}, K}^{\vee \mathfrak{h}, L}: \mathcal{C}(\mathfrak{g}, K) \rightarrow \mathcal{C}(\mathfrak{h}, L), \quad V \mapsto\left(\operatorname{Hom}_{R(\mathfrak{g}, K)}(R(\mathfrak{g}, K), V)\right)_{L}
$$

For an $(\mathfrak{h}, L)$-module $V$, the $(\mathfrak{g}, K)$-modules $\left(P_{\mathfrak{h}, L}^{\mathfrak{g}, K}\right)_{j}(V)$ and $\left(I_{\mathfrak{h}, L}^{\mathfrak{g}, K}\right)^{j}(V)$ are called cohomologically induced modules.

\section{§3. O-modules associated with $(\mathfrak{g}, K)$-modules}

Let $G$ be a complex linear algebraic group acting on a variety (or more generally a scheme) $X$. Let $a: G \times X \rightarrow X$ be the action map and $p_{2}: G \times X \rightarrow X$ the second projection. Write $\mathcal{O}_{X}$ for the structure sheaf of $X$ and $a^{*}, p_{2}^{*}$ for the inverse image functors as $\mathcal{O}$-modules. We say that an $\mathcal{O}_{X}$-module $\mathcal{M}$ is $G$-equivariant if there is an isomorphism $a^{*} \mathcal{M} \simeq p_{2}^{*} \mathcal{M}$ satisfying the cocycle condition. For a $G$-equivariant $\mathcal{O}_{X}$-module $\mathcal{M}$, the $G$-action on $\mathcal{M}$ differentiates to a $\mathfrak{g}$-action on $\mathcal{M}$. 
Definition 3.1. Suppose that $H$ is a closed algebraic subgroup of $G$, and $X=$ $G / H$ is the quotient variety. For an algebraic $H$-module $V$, define $\mathcal{V}_{X}$ as the $G$ equivariant quasi-coherent $\mathcal{O}_{X}$-module that has typical fiber $V$.

The category of $G$-equivariant quasi-coherent $\mathcal{O}_{X}$-modules is equivalent to the category of algebraic $H$-modules, and $\mathcal{V}_{X}$ is the $\mathcal{O}_{X}$-module which corresponds to $V$ via this equivalence. It also corresponds to the associated bundle $G \times_{H} V \rightarrow G / H$. The local sections of $\mathcal{V}_{X}$ can be identified with the $V$-valued regular functions $f$ on open subsets of $G$ satisfying $f(g h)=h^{-1} \cdot f(g)$ for $h \in H$. We often use this identification in the following.

Note that $\mathcal{V}_{X}$ is locally free if $V$ is finite-dimensional. Indeed, let $v_{1}, \ldots, v_{n}$ be a basis of $V$ and take local sections $\widetilde{v}_{1}, \ldots, \widetilde{v}_{n}$ such that $\widetilde{v}_{i}(e)=v_{i}$ for the identity element $e \in G$. Then the map $\mathcal{O}_{X}^{\oplus n} \rightarrow \mathcal{V}_{X}$ given by $\left(f_{i}\right)_{i} \mapsto \sum_{i=1}^{n} f_{i} \widetilde{v}_{i}$ is defined near the base point $e H \in G / H$ and is an isomorphism on some open neighborhood of $e H$.

Suppose that $X$ is a smooth $G$-variety. Then the infinitesimal action is defined as a Lie algebra homomorphism from the Lie algebra $\mathfrak{g}$ of $G$ to the space of vector fields $\mathcal{T}(X)$ on $X$. Denote the image of $\xi \in \mathfrak{g}$ by $\xi_{X} \in \mathcal{T}(X)$. Then $\xi_{X}$ gives a first-order differential operator on the structure sheaf $\mathcal{O}_{X}$. Let $\widetilde{\mathfrak{g}}_{X}:=\mathcal{O}_{X} \otimes_{\mathbb{C}} \mathfrak{g}$. This module becomes a Lie algebroid in a natural way (see [BB93, §1.2]): the Lie bracket is defined by

$$
[f \otimes \xi, g \otimes \eta]=f g \otimes[\xi, \eta]+f \xi_{X}(g) \otimes \eta-g \eta_{X}(f) \otimes \xi
$$

for $f, g \in \mathcal{O}_{X}$ and $\xi, \eta \in \mathfrak{g}$. Here $f \in \mathcal{O}_{X}$ means that $f$ is a local section of $\mathcal{O}_{X}$. Similar notation will be used for other sheaves. Write $U\left(\widetilde{\mathfrak{g}}_{X}\right)\left(\simeq \mathcal{O}_{X} \otimes U(\mathfrak{g})\right)$ for the universal enveloping algebra of $\widetilde{\mathfrak{g}}_{X}$. Then a $U\left(\widetilde{\mathfrak{g}}_{X}\right)$-module is identified with an $\mathcal{O}_{X}$-module $\mathcal{M}$ with a $\mathfrak{g}$-action satisfying $\xi(f m)=\xi_{X}(f) m+f(\xi m)$ for $\xi \in \mathfrak{g}$, $f \in \mathcal{O}_{X}$, and $m \in \mathcal{M}$.

Let $\mathcal{T}_{X}$ be the tangent sheaf of $X$ and let $p: \tilde{\mathfrak{g}}_{X}\left(=\mathcal{O}_{X} \otimes_{\mathbb{C}} \mathfrak{g}\right) \rightarrow \mathcal{T}_{X}$ be the map given by $f \otimes \xi \mapsto f \xi_{X}$. Then the kernel $\mathcal{H}:=\operatorname{ker} p$ is isomorphic to the $G$-equivariant locally free $\mathcal{O}_{X}$-module with typical fiber $\mathfrak{h}$. Let $\mathcal{D}_{X}$ be the ring of differential operators on $X$. The map $p$ extends to $p: U\left(\widetilde{\mathfrak{g}}_{X}\right) \rightarrow \mathcal{D}_{X}$ and descends to an isomorphism of algebras

$$
U\left(\widetilde{\mathfrak{g}}_{X}\right) / U\left(\widetilde{\mathfrak{g}}_{X}\right) \mathcal{H} \stackrel{\sim}{\longrightarrow} \mathcal{D}_{X}
$$

We will work in the following setting.

Setting 3.2. Let $i: K \rightarrow G$ be a homomorphism of complex linear algebraic groups with finite kernel. Let $H$ be a closed algebraic subgroup of $G$. Put $M:=$ 
$i^{-1}(H)$, which is an algebraic subgroup of $K$, and write $X:=G / H$ and $Y:=K / M$ for the quotient varieties. The map $i: K \rightarrow G$ induces an injective morphism $i: Y \rightarrow X$ between the quotient varieties and an injective homomorphism $d i$ : $\mathfrak{k} \rightarrow \mathfrak{g}$ between Lie algebras. We identify $\mathfrak{k}$ with its image $\operatorname{di}(\mathfrak{k})$ and regard $\mathfrak{k}$ as a subalgebra of $\mathfrak{g}$.

In particular, $(\mathfrak{g}, K)$ and $(\mathfrak{h}, M)$ become pairs in the sense of Definition 2.1, where $\mathfrak{h}$ is the Lie algebra of $H$.

Let $e \in K$ be the identity element and let $o:=e M \in Y$ be the base point of $Y$. Write

$$
\mathcal{I}_{Y}:=\left\{f \in \mathcal{O}_{X}: f(y)=0 \text { for } y \in Y\right\}, \quad \mathcal{I}_{o}:=\left\{f \in \mathcal{O}_{X}: f(o)=0\right\},
$$

so $\mathcal{I}_{Y}$ is the defining ideal of the closure $\bar{Y}$ of $Y$. It follows that $i^{-1} \mathcal{O}_{X} / \mathcal{I}_{Y} \simeq \mathcal{O}_{Y}$. Here $i^{-1}$ denotes the inverse image functor for the sheaves of abelian groups. For an $i^{-1} \mathcal{O}_{X}$-module $\mathcal{M}$, the support of the sheaf $\mathcal{M} /\left(i^{-1} \mathcal{I}_{o}\right) \mathcal{M}$ is contained in $\{o\}$ so it is regarded as a vector space.

Let $Y_{p}$ be the scheme $\left(Y, i^{-1} \mathcal{O}_{X} /\left(\mathcal{I}_{Y}\right)^{p}\right)$ for $p \geq 1$. If locally we have $X=$ $\operatorname{Spec} A, Y=\operatorname{Spec} I$, and $Y$ is closed in $X$, then $Y_{p}=\operatorname{Spec}\left(A / I^{p}\right)$. The scheme $Y_{1}$ is identified with the algebraic variety $Y$. If $\mathcal{M}$ is an $i^{-1} \mathcal{O}_{X}$-module, then the sheaf $\mathcal{M} /\left(i^{-1} \mathcal{I}_{Y}\right)^{p} \mathcal{M}$ can be viewed as an $\mathcal{O}_{Y_{p}}$-module.

The inverse image $i^{-1} U\left(\widetilde{\mathfrak{g}}_{X}\right)$ of $U\left(\widetilde{\mathfrak{g}}_{X}\right)$ is a sheaf of algebras on $Y$ and an $i^{-1} \mathcal{O}_{X}$-bimodule. We will call $i^{-1} U\left(\widetilde{\mathfrak{g}}_{X}\right)$-modules simply $i^{-1} \widetilde{\mathfrak{g}}_{X}$-modules. The $K$ action on $i^{-1} \widetilde{\mathfrak{g}}_{X}$ is given by $f \otimes \xi \mapsto(k \cdot f) \otimes \operatorname{Ad}(i(k))(\xi)$ for $f \in i^{-1} \mathcal{O}_{X}, \xi \in \mathfrak{g}$, $k \in K$. Suppose that $\mathcal{M}$ is an $i^{-1} \widetilde{\mathfrak{g}}_{X}$-module and let $i^{-1} \widetilde{\mathfrak{g}}_{X} \otimes \mathcal{M} \rightarrow \mathcal{M}$ be the action map. Then the inclusion $\mathfrak{g} \cdot\left(\mathcal{I}_{Y}\right)^{p} \subset\left(\mathcal{I}_{Y}\right)^{p-1}$ induces a map $i^{-1} \widetilde{\mathfrak{g}}_{X} \otimes$ $\mathcal{M} /\left(i^{-1} \mathcal{I}_{Y}\right)^{p} \mathcal{M} \rightarrow \mathcal{M} /\left(i^{-1} \mathcal{I}_{Y}\right)^{p-1} \mathcal{M}$. The $K$-actions on $X$ and $Y$ induce a $K$ action on $Y_{p}$. Since $Y$ is $K$-stable in $X$, we have $\mathfrak{k} \cdot\left(\mathcal{I}_{Y}\right)^{p} \subset\left(\mathcal{I}_{Y}\right)^{p}$. Therefore, we can define a $\mathfrak{k}$-action on $\mathcal{M} /\left(i^{-1} \mathcal{I}_{Y}\right)^{p} \mathcal{M}$. Similarly, we have $\mathfrak{h} \cdot \mathcal{I}_{o} \subset \mathcal{I}_{o}$ and we can equip $\mathcal{M} /\left(i^{-1} \mathcal{I}_{o}\right) \mathcal{M}$ with an $\mathfrak{h}$-module structure.

Definition 3.3. Let $V$ be an $(\mathfrak{h}, M)$-module. We say an $i^{-1} \widetilde{\mathfrak{g}}_{X}$-module $\mathcal{V}$ is associated with $V$ if $\mathcal{V} /\left(i^{-1} \mathcal{I}_{Y}\right)^{p} \mathcal{V}$ is a $K$-equivariant quasi-coherent $\mathcal{O}_{Y_{p}}$-module for all $p \geq 1$ and the following five assumptions hold.

(1) The canonical map

$$
\mathcal{V} /\left(i^{-1} \mathcal{I}_{Y}\right)^{p} \mathcal{V} \rightarrow \mathcal{V} /\left(i^{-1} \mathcal{I}_{Y}\right)^{p-1} \mathcal{V}
$$

commutes with $K$-actions for $p \geq 2$.

(2) $\mathcal{V} /\left(i^{-1} \mathcal{I}_{Y}\right)^{p} \mathcal{V}$ is a flat $\mathcal{O}_{Y_{p}}$-module for $p \geq 1$. 
(3) The action map $i^{-1} \widetilde{\mathfrak{g}}_{X} \otimes \mathcal{V} /\left(i^{-1} \mathcal{I}_{Y}\right)^{p} \mathcal{V} \rightarrow \mathcal{V} /\left(i^{-1} \mathcal{I}_{Y}\right)^{p-1} \mathcal{V}$ commutes with $K$-actions for $p \geq 2$. Here $K$ acts on $i^{-1} \widetilde{\mathfrak{g}}_{X} \otimes \mathcal{V} /\left(i^{-1} \mathcal{I}_{Y}\right)^{p} \mathcal{V}$ diagonally.

(4) The $\mathfrak{k}$-action on $\mathcal{V} /\left(i^{-1} \mathcal{I}_{Y}\right)^{p} \mathcal{V}$ induced from the $\mathfrak{g}$-action on $\mathcal{V}$ coincides with the differential of the $K$-action on $\mathcal{V} /\left(i^{-1} \mathcal{I}_{Y}\right)^{p} \mathcal{V}$ for $p \geq 1$.

(5) There is an isomorphism $\iota: \mathcal{V} /\left(i^{-1} \mathcal{I}_{o}\right) \mathcal{V} \stackrel{\sim}{\rightarrow} V$ which commutes with $\mathfrak{h}$-actions and $M$-actions.

Remark 3.4. The $\mathfrak{g}$-action and the $K$-action on $\mathcal{V}$ induce an $\mathfrak{h}$-action and an $M$ action on $\mathcal{V} /\left(i^{-1} \mathcal{I}_{o}\right) \mathcal{V}$. The conditions (3) and (4) imply that $\mathcal{V} /\left(i^{-1} \mathcal{I}_{o}\right) \mathcal{V}$ becomes an $(\mathfrak{h}, M)$-module.

Example 3.5. Suppose that $V$ is an $H$-module and define the $G$-equivariant quasi-coherent $\mathcal{O}_{X}$-module $\mathcal{V}_{X}$ as in Definition 3.1. The $G$-action on $\mathcal{V}_{X}$ induces a $\mathfrak{g}$-action and a $K$-action on $\mathcal{V}_{X}$. Then by regarding $V$ as an (h, $\left.M\right)$-module, $i^{-1} \mathcal{V}_{X}$ is associated with $V$.

We will construct an $i^{-1} \widetilde{\mathfrak{g}}_{X}$-module associated with an arbitrary $(\mathfrak{h}, M)$ module in Section 5.

Example 3.6. Let $\mathcal{V}$ and $\mathcal{W}$ be $i^{-1} \widetilde{\mathfrak{g}}_{X}$-modules associated with $(\mathfrak{h}, M)$-modules $V$ and $W$, respectively. Then the tensor product $\mathcal{V} \otimes_{i^{-1} \mathcal{O}_{X}} \mathcal{W}$ is associated with the $(\mathfrak{h}, M)$-module $V \otimes W$.

We can define the pull-back of $i^{-1} \widetilde{\mathfrak{g}}_{X}$-modules associated with $V$ in the following way. Let $K^{\prime}, G^{\prime}, H^{\prime}$ be another triple of algebraic groups satisfying the assumptions in Setting 3.2. In particular, the map $i^{\prime}: K^{\prime} \rightarrow G^{\prime}$ induces a morphism of the quotient varieties $i^{\prime}: K^{\prime} / M^{\prime} \rightarrow G^{\prime} / H^{\prime}$, where $M^{\prime}:=\left(i^{\prime}\right)^{-1}\left(H^{\prime}\right)$. Suppose that $\varphi_{K}: K^{\prime} \rightarrow K$ and $\varphi: G^{\prime} \rightarrow G$ are homomorphisms such that the diagram

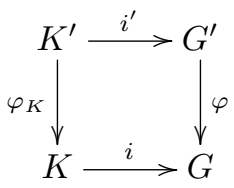

commutes and that $\varphi\left(H^{\prime}\right) \subset H$. Then $\varphi_{K}\left(M^{\prime}\right) \subset M$. The maps $\varphi, \varphi_{K}$ induce morphisms $\varphi: X^{\prime}:=G^{\prime} / H^{\prime} \rightarrow X, \varphi_{K}: Y^{\prime}:=K^{\prime} / M^{\prime} \rightarrow Y$ and $\varphi_{p}: Y_{p}^{\prime}:=$ $\left(Y^{\prime},\left(i^{\prime}\right)^{-1} \mathcal{O}_{X^{\prime}} /\left(\mathcal{I}_{Y^{\prime}}\right)^{p}\right) \rightarrow Y_{p}$. We get the commutative diagram

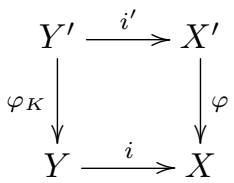


Suppose that $\mathcal{V}$ is an $i^{-1} \widetilde{\mathfrak{g}}_{X}$-module associated with an $(\mathfrak{h}, M)$-module $V$. Let $\mathcal{V}^{\prime}:=\left(i^{\prime}\right)^{-1} \mathcal{O}_{X^{\prime}} \otimes_{\left(\varphi \circ i^{\prime}\right)^{-1} \mathcal{O}_{X}} \varphi_{K}^{-1} \mathcal{V}$. We define a $\mathfrak{g}^{\prime}$-action on $\mathcal{V}^{\prime}$ by $\xi(f \otimes v)=$ $\xi_{X^{\prime}}(f) \otimes v+f \otimes \varphi(\xi) v$ for $\xi \in \mathfrak{g}^{\prime}, f \in\left(i^{\prime}\right)^{-1} \mathcal{O}_{X^{\prime}}$, and $v \in \varphi_{K}^{-1} \mathcal{V}$ so that $\mathcal{V}^{\prime}$ becomes an $\left(i^{\prime}\right)^{-1} \widetilde{\mathfrak{g}^{\prime}}{ }^{\prime}$, module. Since

$$
\mathcal{V}^{\prime} /\left(\left(i^{\prime}\right)^{-1} \mathcal{I}_{Y^{\prime}}\right)^{p} \mathcal{V}^{\prime} \simeq\left(i^{\prime}\right)^{-1} \mathcal{O}_{X^{\prime}} /\left(\mathcal{I}_{Y^{\prime}}\right)^{p} \otimes_{\left(\varphi \circ i^{\prime}\right)^{-1} \mathcal{O}_{X}} \varphi_{K}^{-1} \mathcal{V} \simeq \varphi_{p}^{*}\left(\mathcal{V} /\left(i^{-1} \mathcal{I}_{Y}\right)^{p} \mathcal{V}\right)
$$

the sheaf $\mathcal{V}^{\prime} /\left(\left(i^{\prime}\right)^{-1} \mathcal{I}_{Y^{\prime}}\right)^{p} \mathcal{V}^{\prime}$ is a $K^{\prime}$-equivariant quasi-coherent $\mathcal{O}_{Y_{p}^{\prime}}$-module. We can easily show the following proposition by checking the five assumptions in Definition 3.3.

Proposition 3.7. Let $V$ be an $(\mathfrak{h}, M)$-module and $\mathcal{V}$ an $i^{-1} \widetilde{\mathfrak{g}}_{X}$-module associated with $V$. Then the $\left(i^{\prime}\right)^{-1} \widetilde{\mathfrak{g}}_{X^{\prime}}$-module $\left(i^{\prime}\right)^{-1} \mathcal{O}_{X^{\prime}} \otimes_{\left(\varphi \circ i^{\prime}\right)^{-1} \mathcal{O}_{X}} \varphi_{K}^{-1} \mathcal{V}$ is associated with the $\left(\mathfrak{h}^{\prime}, M^{\prime}\right)$-module $\operatorname{For}_{\mathfrak{h}, M}^{\mathfrak{h}^{\prime}, M^{\prime}}(V)$.

\section{$\S 4$. Localization of cohomological induction}

We retain Setting 3.2. In this section, we assume moreover that $K$ is reductive. Let $M=L \ltimes U$ be a Levi decomposition of $M$, where $L$ is a maximal reductive subgroup of $M$, and $U$ is the unipotent radical of $M$. The corresponding decomposition of the Lie algebra is $\mathfrak{m}=\mathfrak{l} \oplus \mathfrak{u}$.

Let $V$ be an $(\mathfrak{h}, M)$-module. We can view $V$ as an $(\mathfrak{h}, L)$-module by restriction and then define the cohomologically induced module $\left(P_{\mathfrak{h}, L}^{\mathfrak{g}, K}\right)_{j}(V)$ as in Section 2.

In order to state the main theorem, we need a shift of modules by a character (or an invertible sheaf) that we will define in the following. Write $\bigwedge^{\text {top }}(\mathfrak{k} / \mathfrak{l})$ for the top exterior product of $\mathfrak{k} / \mathfrak{l}$ and view it as a one-dimensional $L$-module by the adjoint action. Since $K$ and $L$ are reductive, the identity component of $L$ acts trivially on $\bigwedge^{\text {top }}(\mathfrak{k} / \mathfrak{l})$. We extend the $L$-action on $\bigwedge^{\text {top }}(\mathfrak{k} / \mathfrak{l})$ to an $M$-action by letting $U$ act trivially. Define $\mathcal{L}$ as the $K$-equivariant locally free $\mathcal{O}_{Y}$-module on $Y:=K / M$ whose typical fiber is isomorphic to the $M$-module $\bigwedge^{\text {top }}(\mathfrak{k} / \mathfrak{l})$. The $K$-action on $\mathcal{L}$ differentiates to a $\mathfrak{k}$-action. Then $\mathcal{L}$ becomes a $U\left(\widetilde{\mathfrak{k}}_{Y}\right)$-module and the kernel of the map $\widetilde{\mathfrak{k}}_{Y} \rightarrow \mathcal{T}_{Y}$ acts by zero because the identity component of $M$ acts trivially on $\bigwedge^{\text {top }}(\mathfrak{k} / \mathfrak{l})$. Therefore, $\mathcal{L}$ has a structure of left $\mathcal{D}_{Y}$-module via the isomorphism (3.1) for $Y$.

Let $\mathcal{M}$ be a left $\mathcal{D}_{Y}$-module. Recall that the direct image of $\mathcal{M}$ by $i$ in the category of left $\mathcal{D}$-modules is defined as

$$
i_{+} \mathcal{M}:=i_{*}\left(\left(\mathcal{M} \otimes_{\mathcal{O}_{Y}} \Omega_{Y}\right) \otimes_{\mathcal{D}_{Y}} i^{*} \mathcal{D}_{X}\right) \otimes_{\mathcal{O}_{X}} \Omega_{X}^{\vee},
$$

where $i_{*}$ is the direct image functor for sheaves of abelian groups, $\Omega_{Y}$ is the canonical sheaf of $Y$, and $\Omega_{X}^{\vee}$ is the dual of the canonical sheaf of $X$. Via the map 
$p: U\left(\widetilde{\mathfrak{g}}_{X}\right) \rightarrow \mathcal{D}_{X}$, we can view $i_{+} \mathcal{M}$ as a $\widetilde{\mathfrak{g}}_{X}$-module. The inverse image $i^{-1} i_{+} \mathcal{M}$ as a sheaf of abelian groups is

$$
i^{-1} i_{+} \mathcal{M}=\left(\mathcal{M} \otimes_{\mathcal{O}_{Y}} \Omega_{Y}\right) \otimes_{\mathcal{D}_{Y}} i^{*} \mathcal{D}_{X} \otimes_{i^{-1} \mathcal{O}_{X}} i^{-1} \Omega_{X}^{\vee}
$$

which has an $i^{-1} \widetilde{\mathfrak{g}}_{X}$-module structure. We note that the functor $i^{-1} i_{+}$is exact.

Define subsheaves of $\mathcal{D}_{X}$ by

$$
F_{p} \mathcal{D}_{X}:=\left\{D \in \mathcal{D}_{X}: D\left(\mathcal{I}_{Y}\right)^{p+1} \subset \mathcal{I}_{Y}\right\}
$$

for $p \geq 0$. They are $\mathcal{O}_{X}$-bi-submodules of $\mathcal{D}_{X}$ and form a filtration of $\mathcal{D}_{X}$. It induces a filtration of $i^{-1} i_{+} \mathcal{L}$ :

$$
F_{p} i^{-1} i_{+} \mathcal{L}:=\left(\mathcal{L} \otimes_{\mathcal{O}_{Y}} \Omega_{Y}\right) \otimes_{\mathcal{D}_{Y}} i^{*} F_{p} \mathcal{D}_{X} \otimes_{i^{-1} \mathcal{O}_{X}} i^{-1} \Omega_{X}^{\vee}
$$

It follows from the definition of $F_{p} \mathcal{D}_{X}$ that $F_{p} i^{-1} i_{+} \mathcal{L}$ is annihilated by $\left(i^{-1} \mathcal{I}_{Y}\right)^{p+1}$ and hence is regarded as a quasi-coherent $\mathcal{O}_{Y_{p+1}}$-module.

Here is the main theorem of this article:

Theorem 4.1. In Setting 3.2, assume that $K$ is reductive. Let $M=L \ltimes U$ be a Levi decomposition. Suppose that $V$ is an $(\mathfrak{h}, M)$-module and that $\mathcal{V}$ is an $i^{-1} \widetilde{\mathfrak{g}}_{X}-$ module associated with $V$ (Definition 3.3$)$. Then we have an isomorphism of $(\mathfrak{g}, K)$ modules

$$
\mathrm{H}^{s}\left(Y, i^{-1} i_{+} \mathcal{L} \otimes_{i^{-1} \mathcal{O}_{X}} \mathcal{V}\right) \simeq\left(P_{\mathfrak{h}, L}^{\mathfrak{g}, K}\right)_{u-s}\left(V \otimes \bigwedge^{\mathrm{top}}(\mathfrak{g} / \mathfrak{h})\right)
$$

for $s \in \mathbb{N}$ and $u=\operatorname{dim} U$. (See the remark below for the definition of the $(\mathfrak{g}, K)$ action on the left side.)

Remark 4.2. Since $i^{-1} i_{+} \mathcal{L}$ and $\mathcal{V}$ have $i^{-1} \widetilde{\mathfrak{g}}_{X}$-module structures, the tensor product $i^{-1} i_{+} \mathcal{L} \otimes_{i^{-1} \mathcal{O}_{X}} \mathcal{V}$ becomes an $i^{-1} \widetilde{\mathfrak{g}}_{X}$-module. This gives a $\mathfrak{g}$-action on the cohomology group $\mathrm{H}^{s}\left(Y, i^{-1} i_{+} \mathcal{L} \otimes_{i^{-1} \mathcal{O}_{X}} \mathcal{V}\right)$. In order to define a $K$-action, we use the filtration $F_{p} i^{-1} i_{+} \mathcal{L}$ defined above. By definition, $\left(i^{-1} \mathcal{I}_{Y}\right)^{p+1}$ annihilates $F_{p} i^{-1} i_{+} \mathcal{L}$ and hence

$$
F_{p} i^{-1} i_{+} \mathcal{L} \otimes_{i^{-1} \mathcal{O}_{X}} \mathcal{V} \simeq F_{p} i^{-1} i_{+} \mathcal{L} \otimes_{\mathcal{O}_{Y_{q}}} \mathcal{V} /\left(i^{-1} \mathcal{I}_{Y}\right)^{q} \mathcal{V}
$$

for $p<q$. Since $\mathcal{V} /\left(i^{-1} \mathcal{I}_{Y}\right)^{q} \mathcal{V}$ is a flat $\mathcal{O}_{Y_{q}}$-module by Definition 3.3(2), the map

$$
F_{p-1} i^{-1} i_{+} \mathcal{L} \otimes_{i^{-1} \mathcal{O}_{X}} \mathcal{V} \rightarrow F_{p} i^{-1} i_{+} \mathcal{L} \otimes_{i^{-1} \mathcal{O}_{X}} \mathcal{V}
$$

is injective. We let $K$ act diagonally on the right side of (4.2). This gives a $K$-action on $\mathrm{H}^{s}\left(Y, F_{p} i^{-1} i_{+} \mathcal{L} \otimes_{i^{-1} \mathcal{O}_{X}} \mathcal{V}\right)$. Using the isomorphisms 


$$
\begin{aligned}
\mathrm{H}^{s}\left(Y, i^{-1} i_{+} \mathcal{L}\right. & \left.\otimes_{i^{-1} \mathcal{O}_{X}} \mathcal{V}\right) \simeq \mathrm{H}^{s}\left(Y, \underset{p}{\left.\left(\underset{p}{\lim } F_{p} i^{-1} i_{+} \mathcal{L}\right) \otimes_{i^{-1} \mathcal{O}_{X}} \mathcal{V}\right)}\right. \\
& \simeq \mathrm{H}^{s}\left(Y, \underset{p}{\lim }\left(F_{p} i^{-1} i_{+} \mathcal{L} \otimes_{i^{-1} \mathcal{O}_{X}} \mathcal{V}\right)\right) \simeq \underset{p}{\lim } \mathrm{H}^{s}\left(Y, F_{p} i^{-1} i_{+} \mathcal{L} \otimes_{i^{-1} \mathcal{O}_{X}} \mathcal{V}\right)
\end{aligned}
$$

we define a $K$-action on $\mathrm{H}^{s}\left(Y, i^{-1} i_{+} \mathcal{L} \otimes_{i^{-1} \mathcal{O}_{X}} \mathcal{V}\right)$. With these actions, $\mathrm{H}^{s}\left(Y, i^{-1} i_{+} \mathcal{L}\right.$ $\left.\otimes_{i^{-1} \mathcal{O}_{X}} \mathcal{V}\right)$ becomes a $(\mathfrak{g}, K)$-module because of Definition 3.3(3), (4).

Proof of Theorem 4.1. Let $\widetilde{X}:=G / L$ and $\tilde{Y}:=K / L$ be the quotient varieties. We have the commutative diagram

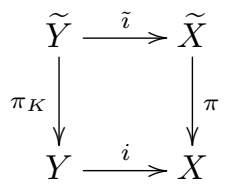

where the maps are defined canonically.

The direct image functor $i_{+}$defined as in (4.1) induces the direct image functor between the bounded derived categories of left $\mathcal{D}$-modules, which we denote by $i_{+}: \mathbf{D}^{\mathrm{b}}\left(\mathcal{D}_{Y}\right) \rightarrow \mathbf{D}^{\mathrm{b}}\left(\mathcal{D}_{X}\right)$. Similarly for $\tilde{\imath}_{+}, \pi_{+}$, and $\left(\pi_{K}\right)_{+}$. We have $\pi_{+} \circ \tilde{\imath}_{+} \simeq$ $i_{+} \circ\left(\pi_{K}\right)_{+}$. Since $\pi_{K}$ is a smooth morphism and the fiber is isomorphic to the affine space $\mathbb{C}^{u}$, it follows that $\left(\pi_{K}\right)_{+} \Omega_{\widetilde{Y}}^{\vee} \simeq \mathcal{L}[u]$ (see [HMSW87]). Here $\mathcal{L}[u] \in \mathbf{D}^{\mathrm{b}}\left(\mathcal{D}_{Y}\right)$ is the complex $(\cdots \rightarrow 0 \rightarrow \mathcal{L} \rightarrow 0 \rightarrow \cdots)$, concentrated in degree $-u$. Therefore, $i_{+}\left(\pi_{K}\right)_{+} \Omega_{\widetilde{Y}}^{\vee} \simeq i_{+} \mathcal{L}[u]$ in $\mathbf{D}^{\mathrm{b}}\left(\mathcal{D}_{X}\right)$.

Since $L$ is reductive, the varieties $\widetilde{X}$ and $\widetilde{Y}$ are affine by Matsushima's criterion. Hence the functor $\tilde{\imath}_{+}$is exact for quasi-coherent $\mathcal{D}$-modules and $\pi_{*}$ is exact for quasi-coherent $\mathcal{O}$-modules.

Denote by $\mathcal{T}_{\tilde{X} / X}$ the sheaf of local vector fields on $\widetilde{X}$ tangent to the fiber of $\pi$, and denote by $\Omega_{\tilde{X} / X}$ the top exterior product of its dual $\mathcal{T}_{\tilde{X} / X}^{\vee}$. We note that there is a natural isomorphism $\Omega_{\tilde{X} / X} \simeq \Omega_{\tilde{X}} \otimes_{\mathcal{O}_{\widetilde{X}}} \pi^{*} \Omega_{X}^{\vee}$. Recall that for $\mathcal{M} \in \mathbf{D}^{\mathrm{b}}\left(\mathcal{D}_{\tilde{X}}\right)$ the direct image $\pi_{+} \mathcal{M}$ is defined as

$$
\pi_{+} \mathcal{M}=\pi_{*}\left(\left(\mathcal{M} \otimes_{\mathcal{O}_{\widetilde{X}}} \Omega_{\widetilde{X}}\right) \otimes_{\mathcal{D}_{\widetilde{X}}}^{\mathbb{L}} \pi^{*} \mathcal{D}_{X}\right) \otimes_{\mathcal{O}_{X}} \Omega_{X}^{\vee}
$$

The left $\mathcal{D}_{\tilde{X}}$-module $\pi^{*} \mathcal{D}_{X}$ has the resolution (see [HMSW87, Appendix A.3.3])

$$
\mathcal{D}_{\widetilde{X}} \otimes_{\mathcal{O}_{\widetilde{X}}} \Lambda^{\bullet} \mathcal{T}_{\widetilde{X} / X} \rightarrow \pi^{*} \mathcal{D}_{X},
$$

where the boundary map $\partial$ on $\mathcal{D}_{\tilde{X}} \otimes_{\mathcal{O}_{\tilde{X}}} \wedge^{\bullet} \mathcal{T}_{\widetilde{X} / X}$ is given as

$$
\begin{aligned}
& D \otimes \widetilde{\xi}_{1} \wedge \cdots \wedge \widetilde{\xi}_{d} \mapsto \sum_{i=1}^{d}(-1)^{i+1} D \widetilde{\xi}_{i} \otimes \widetilde{\xi}_{1} \wedge \cdots \wedge \widetilde{\widetilde{\xi}}_{i} \wedge \cdots \wedge \widetilde{\xi}_{d} \\
& +\sum_{1 \leq i<j \leq d}(-1)^{i+j} D \otimes\left[\widetilde{\xi}_{i}, \widetilde{\xi}_{j}\right] \wedge \widetilde{\xi}_{1} \wedge \cdots \wedge \widehat{\widetilde{\xi}}_{i} \wedge \cdots \wedge \widehat{\widetilde{\xi}}_{j} \wedge \cdots \wedge \widetilde{\xi}_{d}
\end{aligned}
$$


The right $\pi^{-1} \mathcal{D}_{X}$-module structure is not canonically defined on the complex, but the $\mathfrak{g}$-action can be described as

$$
\xi\left(D \otimes \widetilde{\xi}_{1} \wedge \cdots \wedge \widetilde{\xi}_{d}\right)=-D \xi_{\widetilde{X}} \otimes \widetilde{\xi}_{1} \wedge \cdots \wedge \widetilde{\xi}_{d}+D \otimes \xi\left(\widetilde{\xi}_{1} \wedge \cdots \wedge \widetilde{\xi}_{d}\right)
$$

for $\xi \in \mathfrak{g}$. Here we use the $\mathfrak{g}$-action on $\bigwedge \mathcal{T}_{\widetilde{X} / X}$ induced from the $G$-equivariant structure.

By using the resolution (4.3), the direct image $\pi_{+} \tilde{l}_{+} \Omega_{\widetilde{Y}}^{\vee}$ is given as the complex

$$
\pi_{*}\left(\tilde{\imath}_{+} \Omega_{\widetilde{Y}}^{\vee} \otimes_{\mathcal{O}_{\widetilde{X}}} \Omega_{\widetilde{X}} \otimes_{\mathcal{O}_{\widetilde{X}}} \wedge^{\bullet} \mathcal{T}_{\widetilde{X} / X}\right) \otimes_{\mathcal{O}_{X}} \Omega_{X}^{\vee}
$$

As a result, we have

$$
i_{+} \mathcal{L}[u] \simeq \pi_{*}\left(\tilde{\imath}_{+} \Omega_{\widetilde{Y}}^{\vee} \otimes_{\mathcal{O}_{\widetilde{X}}} \wedge^{\bullet} \mathcal{T}_{\widetilde{X} / X} \otimes_{\mathcal{O}_{\widetilde{X}}} \Omega_{\widetilde{X} / X}\right)
$$

and hence

$$
\begin{aligned}
i^{-1} i_{+} \mathcal{L}[u] & \simeq i^{-1} \pi_{*}\left(\tilde{\imath}_{+} \Omega_{\widetilde{Y}}^{\vee} \otimes_{\mathcal{O}_{\widetilde{X}}} \bigwedge^{\bullet} \mathcal{T}_{\widetilde{X} / X} \otimes_{\mathcal{O}_{\widetilde{X}}} \Omega_{\widetilde{X} / X}\right) \\
& \simeq i^{-1} \pi_{*} \tilde{\imath}_{*}\left(\tilde{l}^{-1} \tilde{\imath}_{+} \Omega_{\widetilde{Y}}^{\vee} \otimes_{\tilde{\imath}^{-1} \mathcal{O}_{\widetilde{X}}} \tilde{\imath}^{-1} \bigwedge^{\bullet} \mathcal{T}_{\widetilde{X} / X} \otimes_{\tilde{\imath}^{-1} \mathcal{O}_{\widetilde{X}}} \tilde{\imath}^{-1} \Omega_{\widetilde{X} / X}\right) \\
& \simeq\left(\pi_{K}\right)_{*}\left(\tilde{l}^{-1} \tilde{\imath}_{+} \Omega_{\widetilde{Y}}^{\vee} \otimes_{\tilde{i}^{-1} \mathcal{O}_{\widetilde{X}}} \tilde{\imath}^{-1}\left(\bigwedge^{\bullet} \mathcal{T}_{\widetilde{X} / X} \otimes_{\mathcal{O}_{\widetilde{X}}} \Omega_{\widetilde{X} / X}\right)\right)
\end{aligned}
$$

There is a natural morphism of complexes of $i^{-1} \mathcal{O}_{X^{-}}$modules

$$
\begin{aligned}
& \psi:\left(\pi_{K}\right)_{*}\left(\tilde{\imath}^{-1} \tilde{\imath}_{+} \Omega_{\widetilde{Y}}^{\vee} \otimes_{\tilde{\imath}^{-1} \mathcal{O}_{\widetilde{X}}} \tilde{\imath}^{-1}\left(\bigwedge^{\bullet} \mathcal{T}_{\widetilde{X} / X} \otimes_{\mathcal{O}_{\widetilde{X}}} \Omega_{\widetilde{X} / X}\right)\right) \otimes_{i^{-1} \mathcal{O}_{X}} \mathcal{V} \\
& \rightarrow\left(\pi_{K}\right)_{*}\left(\tilde{\imath}^{-1} \tilde{\imath}_{+} \Omega_{\widetilde{Y}}^{\vee} \otimes_{\tilde{\imath}^{-1} \mathcal{O}_{\widetilde{X}}} \tilde{\imath}^{-1}\left(\bigwedge^{\bullet} \mathcal{T}_{\widetilde{X} / X} \otimes_{\mathcal{O}_{\widetilde{X}}} \Omega_{\widetilde{X} / X}\right) \otimes_{\pi_{K}^{-1} i^{-1} \mathcal{O}_{X}} \pi_{K}^{-1} \mathcal{V}\right)
\end{aligned}
$$

We claim that $\psi$ is an isomorphism. Indeed, if $F_{p} \tilde{\imath}^{-1} \tilde{\imath}_{+} \Omega_{\widetilde{Y}}^{\vee}$ denotes the filtration of $\tilde{\imath}^{-1} \tilde{\imath}_{+} \Omega_{\widetilde{Y}}^{\vee}$ defined in a way similar to $F_{p} i^{-1} i_{+} \mathcal{L}$, then we get a map

$$
\begin{aligned}
\psi_{p}:\left(\pi_{K}\right)_{*}\left(F_{p} \tilde{\imath}^{-1} \tilde{\imath}_{+} \Omega_{\widetilde{Y}}^{\vee} \otimes_{\tilde{\imath}^{-1} \mathcal{O}_{\widetilde{X}}} \tilde{\imath}^{-1}\left(\bigwedge^{\bullet} \mathcal{T}_{\widetilde{X} / X} \otimes_{\mathcal{O}_{\widetilde{X}}} \Omega_{\widetilde{X} / X}\right)\right) \otimes_{i^{-1} \mathcal{O}_{X}} \mathcal{V} \\
\rightarrow\left(\pi_{K}\right)_{*}\left(F_{p} \tilde{\imath}^{-1} \tilde{\imath}_{+} \Omega_{\widetilde{Y}}^{\vee} \otimes_{\tilde{\imath}^{-1} \mathcal{O}_{\widetilde{X}}} \tilde{\imath}^{-1}\left(\bigwedge^{\bullet} \mathcal{T}_{\widetilde{X} / X} \otimes_{\mathcal{O}_{\widetilde{X}}} \Omega_{\widetilde{X} / X}\right) \otimes_{\pi_{K}^{-1} i^{-1} \mathcal{O}_{X}} \pi_{K}^{-1} \mathcal{V}\right) .
\end{aligned}
$$

It is enough to show that $\psi_{p}$ is an isomorphism for all $p \geq 0$ because $\lim _{p} F_{p} \tilde{\imath}^{-1} \tilde{\imath}_{+} \Omega_{\widetilde{Y}}^{\vee}$ $\simeq \tilde{\imath}^{-1} \tilde{\imath}_{+} \Omega_{\widetilde{Y}}^{\vee}$. Since the ideal $\pi_{K}^{-1}\left(i^{-1} \mathcal{I}_{Y}\right)^{p+1}$ of $\pi_{K}^{-1} i^{-1} \mathcal{O}_{X}$ annihilates $F_{p} \tilde{\imath}^{-1} \tilde{\imath}_{+} \Omega_{\widetilde{Y}}^{\vee}$, we have

$$
\begin{aligned}
& \left(\pi_{K}\right)_{*}\left(F_{p} \tilde{\imath}^{-1} \tilde{\imath}_{+} \Omega_{\widetilde{Y}}^{\vee} \otimes_{\tilde{\imath}^{-1} \mathcal{O}_{\widetilde{X}}} \tilde{\imath}^{-1}\left(\bigwedge^{\bullet} \mathcal{T}_{\widetilde{X} / X} \otimes_{\mathcal{O}_{\widetilde{X}}} \Omega_{\widetilde{X} / X}\right)\right) \otimes_{i^{-1} \mathcal{O}_{X}} \mathcal{V} \\
& \simeq\left(\pi_{K}\right)_{*}\left(F_{p} \tilde{\imath}^{-1} \tilde{\imath}_{+} \Omega_{\widetilde{Y}}^{\vee} \otimes_{\tilde{\imath}^{-1} \mathcal{O}_{\widetilde{X}}} \tilde{\imath}^{-1}\left(\bigwedge^{\bullet} \mathcal{T}_{\widetilde{X} / X} \otimes_{\mathcal{O}_{\widetilde{X}}} \Omega_{\widetilde{X} / X}\right)\right) \otimes_{\mathcal{O}_{Y_{p+1}}}\left(\mathcal{V} /\left(i^{-1} \mathcal{I}_{Y}\right)^{p+1} \mathcal{V}\right) .
\end{aligned}
$$

By Definition 3.3(2), $\mathcal{V} /\left(i^{-1} \mathcal{I}_{Y}\right)^{p+1} \mathcal{V}$ is a flat $\mathcal{O}_{Y_{p+1}}$-module. Hence the projection formula shows that $\psi_{p}$ is an isomorphism and the claim is verified.

The successive quotient of the filtration

$$
F_{p} \mathcal{M}:=F_{p} \tilde{\imath}^{-1} \tilde{\imath}_{+} \Omega_{\widetilde{Y}}^{\vee} \otimes_{\tilde{\imath}^{-1} \mathcal{O}_{\widetilde{X}}} \tilde{\imath}^{-1}\left(\bigwedge^{d} \mathcal{T}_{\widetilde{X} / X} \otimes_{\mathcal{O}_{\widetilde{X}}} \Omega_{\widetilde{X} / X}\right) \otimes_{\pi_{K}^{-1} i^{-1} \mathcal{O}_{X}} \pi_{K}^{-1} \mathcal{V}
$$


is

$\left(F_{p} \tilde{\imath}^{-1} \tilde{\imath}_{+} \Omega_{\widetilde{Y}}^{\vee} / F_{p-1} \tilde{\imath}^{-1} \tilde{\imath}_{+} \Omega_{\widetilde{Y}}^{\vee}\right) \otimes_{\mathcal{O}_{\tilde{Y}}} \tilde{\imath}^{*}\left(\bigwedge^{d} \mathcal{T}_{\widetilde{X} / X} \otimes_{\mathcal{O}_{\widetilde{X}}} \Omega_{\widetilde{X} / X}\right) \otimes_{\mathcal{O}_{\tilde{Y}}} \pi_{K}^{*}\left(\mathcal{V} /\left(i^{-1} \mathcal{I}_{Y}\right) \mathcal{V}\right)$,

which is a quasi-coherent $\mathcal{O}_{\tilde{Y}}$-module. Since $\tilde{Y}$ is affine, $\mathrm{H}^{s}\left(\widetilde{Y}, F_{p} \mathcal{M} / F_{p-1} \mathcal{M}\right)=0$ for $s>0$. Hence $\mathrm{H}^{s}\left(\widetilde{Y}, F_{p} \mathcal{M}\right)=0$ and

$$
\mathrm{H}^{s}\left(\widetilde{Y}, \tilde{\imath}^{-1} \tilde{\imath}_{+} \Omega_{\widetilde{Y}}^{\vee} \otimes_{\tilde{\imath}^{-1} \mathcal{O}_{\widetilde{X}}} \tilde{l}^{-1}\left(\bigwedge^{d} \mathcal{T}_{\widetilde{X} / X} \otimes_{\mathcal{O}_{\widetilde{X}}} \Omega_{\widetilde{X} / X}\right) \otimes_{\pi_{K}^{-1} i^{-1} \mathcal{O}_{X}} \pi_{K}^{-1} \mathcal{V}\right)=0
$$

for $s>0$. By (4.4) and (4.5), we conclude that

$$
\begin{aligned}
& \mathrm{H}^{s}\left(Y, i^{-1} i_{+} \mathcal{L} \otimes_{i^{-1} \mathcal{O}_{X}} \mathcal{V}\right) \\
& \quad \simeq \mathrm{H}^{s-u} \Gamma\left(\widetilde{Y}, \tilde{\imath}^{-1} \tilde{\imath}_{+} \Omega_{\widetilde{Y}}^{\vee} \otimes_{\tilde{\imath}^{-1} \mathcal{O}_{\widetilde{X}}} \tilde{\imath}^{-1}\left(\bigwedge^{\bullet} \mathcal{T}_{\tilde{X} / X} \otimes_{\mathcal{O}_{\widetilde{X}}} \Omega_{\tilde{X} / X}\right) \otimes_{\pi_{K}^{-1} i^{-1} \mathcal{O}_{X}} \pi_{K}^{-1} \mathcal{V}\right) .
\end{aligned}
$$

Since $\tilde{\imath}^{-1} \tilde{l}_{+} \Omega_{\widetilde{Y}}^{\vee} \otimes_{\tilde{\imath}^{-1} \mathcal{O}_{\widetilde{X}}} \tilde{\imath}^{-1} \Omega_{\widetilde{X}} \simeq \mathcal{O}_{\widetilde{Y}} \otimes_{\mathcal{D}_{\tilde{Y}}} \tilde{\imath}^{*} \mathcal{D}_{\widetilde{X}}$, we have

$$
\begin{aligned}
\tilde{\imath}^{-1} \tilde{\imath}_{+} \Omega_{\widetilde{Y}}^{\vee} & \otimes_{\tilde{\imath}^{-1} \mathcal{O}_{\widetilde{X}}} \tilde{\imath}^{-1}\left(\bigwedge^{\bullet} \mathcal{T}_{\widetilde{X} / X} \otimes_{\mathcal{O}_{\widetilde{X}}} \Omega_{\tilde{X} / X}\right) \otimes_{\pi_{K}^{-1} i^{-1} \mathcal{O}_{X}} \pi_{K}^{-1} \mathcal{V} \\
& \simeq \mathcal{O}_{\widetilde{Y}} \otimes_{\mathcal{D}_{\tilde{Y}}} \tilde{\imath}^{*} \mathcal{D}_{\widetilde{X}} \otimes_{\tilde{\imath}^{-1} \mathcal{O}_{\widetilde{X}}} \tilde{\imath}^{-1} \Lambda^{\bullet} \mathcal{T}_{\widetilde{X} / X} \otimes_{\pi_{K}^{-1} i^{-1} \mathcal{O}_{X}} \pi_{K}^{-1}\left(\mathcal{V} \otimes_{i^{-1} \mathcal{O}_{X}} i^{-1} \Omega_{X}^{\vee}\right)
\end{aligned}
$$

If we put

$$
\mathcal{V}^{-d}:=\tilde{\imath}^{-1} \bigwedge^{d} \mathcal{T}_{\tilde{X} / X} \otimes_{\pi_{K}^{-1} i^{-1} \mathcal{O}_{X}} \pi_{K}^{-1}\left(\mathcal{V} \otimes_{i^{-1} \mathcal{O}_{X}} i^{-1} \Omega_{X}^{\vee}\right),
$$

then we obtain

$$
\mathrm{H}^{s}\left(Y, i^{-1} i_{+} \mathcal{L} \otimes_{i^{-1} \mathcal{O}_{X}} \mathcal{V}\right) \simeq \mathrm{H}^{s-u} \Gamma\left(\widetilde{Y}, \mathcal{O}_{\widetilde{Y}} \otimes_{\mathcal{D}_{\tilde{Y}}} \tilde{\imath}^{*} \mathcal{D}_{\widetilde{X}} \otimes_{\tilde{\imath}^{-1} \mathcal{O}_{\widetilde{X}}} \mathcal{V}^{\bullet}\right)
$$

The boundary map

$$
\partial: \mathcal{O}_{\tilde{Y}} \otimes_{\mathcal{D}_{\tilde{Y}}} \tilde{l}^{*} \mathcal{D}_{\tilde{X}} \otimes_{\tilde{i}^{-1} \mathcal{O}_{\tilde{X}}} \mathcal{V}^{-d} \rightarrow \mathcal{O}_{\tilde{Y}} \otimes_{\mathcal{D}_{\tilde{Y}}} \tilde{\imath}^{*} \mathcal{D}_{\tilde{X}} \otimes_{\tilde{i}^{-1} \mathcal{O}_{\tilde{X}}} \mathcal{V}^{-d+1}
$$

is given by

$f \otimes D \otimes \widetilde{\xi_{1}} \wedge \cdots \wedge \widetilde{\xi}_{d} \otimes v$

$$
\begin{aligned}
& \mapsto \sum_{i=1}^{d}(-1)^{i+1} f \otimes D \widetilde{\xi}_{i} \otimes \widetilde{\xi}_{1} \wedge \cdots \wedge \widehat{\widetilde{\xi}}_{i} \wedge \cdots \wedge \widetilde{\xi}_{d} \otimes v \\
& \quad+\sum_{1 \leq i<j \leq d}(-1)^{i+j} f \otimes D \otimes\left[\widetilde{\xi}_{i}, \widetilde{\xi}_{j}\right] \wedge \widetilde{\xi}_{1} \wedge \cdots \wedge \widehat{\widetilde{\xi}}_{i} \wedge \cdots \wedge \widehat{\widetilde{\xi}_{j}} \wedge \cdots \wedge \widetilde{\xi}_{d} \otimes v,
\end{aligned}
$$

where $f \in \mathcal{O}_{\tilde{Y}}, D \in \tilde{\imath}^{*} \mathcal{D}_{\tilde{X}}, \widetilde{\xi_{1}}, \ldots, \widetilde{\xi}_{d} \in \tilde{\imath}^{-1} \mathcal{T}_{\tilde{X} / X}$, and $v \in \pi_{K}^{-1}\left(\mathcal{V} \otimes_{i^{-1} \mathcal{O}_{X}} i^{-1} \Omega_{X}^{\vee}\right)$.

The right side of (4.6) can be computed by using the following lemma.

Lemma 4.3. Let $V^{\prime}$ be an $L$-module, or equivalently an $(\mathfrak{l}, L)$-module. Let $\mathcal{V}^{\prime}$ be an $\tilde{\imath}^{-1} \widetilde{\mathfrak{g}}_{\tilde{X}}$-module associated with $V^{\prime}$. Then

$$
\Gamma\left(\widetilde{Y}, \mathcal{O}_{\widetilde{Y}} \otimes_{\mathcal{D}_{\widetilde{Y}}} \tilde{\imath}^{*} \mathcal{D}_{\widetilde{X}} \otimes_{\tilde{\imath}^{-1} \mathcal{O}_{\widetilde{X}}} \mathcal{V}^{\prime}\right) \simeq R(\mathfrak{g}, K) \otimes_{R(L)} V^{\prime}
$$


Proof. The proof is similar to that of [Osh11, Lemma 3.4].

Using the right $\tilde{\imath}^{-1} \mathcal{D}_{\widetilde{X}}$-module structure of $\tilde{\imath}^{*} \mathcal{D}_{\widetilde{X}}$, we can define a $\mathfrak{g}$-action $\rho$ on the sheaf $\tilde{\imath}^{*} \mathcal{D}_{\tilde{X}} \otimes_{\tilde{\imath}^{-1}} \mathcal{O}_{\widetilde{X}} \mathcal{V}^{\prime}$ by

$$
\rho(\xi)(D \otimes v):=-D \xi_{\widetilde{X}} \otimes v+D \otimes \xi v
$$

for $\xi \in \mathfrak{g}, D \in \tilde{\imath}^{*} \mathcal{D}_{\tilde{X}}$, and $v \in \mathcal{V}^{\prime}$. Moreover, the sheaf $\tilde{\imath}^{*} \mathcal{D}_{\widetilde{X}} \otimes_{\tilde{\imath}^{-1}} \mathcal{O}_{\widetilde{X}} \mathcal{V}^{\prime}$ is $K$ equivariant. We denote this $K$-action and also its infinitesimal $\mathfrak{k}$-action by $\nu$. Definition 3.3(4) implies that the $\mathfrak{k}$-action $\nu$ is given by

$$
\nu(\eta)(D \otimes v)=\eta_{\widetilde{Y}} D \otimes v-D \eta_{\widetilde{X}} \otimes v+D \otimes \eta v
$$

for $\eta \in \mathfrak{k}$. Here, $\eta_{\widetilde{Y}} D$ and $D \eta_{\widetilde{X}}$ are defined by the $\left(\mathcal{D}_{\widetilde{Y}}, \tilde{\imath}^{-1} \mathcal{D}_{\widetilde{X}}\right)$-bimodule structure on $\tilde{\imath}^{*} \mathcal{D}_{\tilde{X}}$. Then it follows from Definition 3.3(3) that $\Gamma\left(\widetilde{Y}, \tilde{\imath}^{*} \mathcal{D}_{\widetilde{X}} \otimes_{\tilde{\imath}^{-1}} \mathcal{O}_{\widetilde{X}} \mathcal{V}^{\prime}\right)$ is a weak $(\mathfrak{g}, K)$-module in the sense of [BL95], namely,

$$
\nu(k) \rho(\xi) \nu\left(k^{-1}\right)=\rho(\operatorname{Ad}(i(k)) \xi)
$$

for $k \in K$ and $\xi \in \mathfrak{g}$. Put $\omega(\eta):=\nu(\eta)-\rho(\eta)$ for $\eta \in \mathfrak{k}$. Then $\omega(\eta)$ is given by

$$
\omega(\eta)(D \otimes v)=\eta_{\widetilde{Y}} D \otimes v .
$$

Since $\widetilde{Y}$ is an affine variety, $\Gamma\left(\widetilde{Y}, \mathcal{D}_{\widetilde{Y}}\right)$ is generated by $U(\mathfrak{k})$ and $\mathcal{O}(\widetilde{Y})$ as an algebra. Therefore,

$$
\begin{aligned}
\Gamma\left(\widetilde{Y}, \mathcal{O}_{\widetilde{Y}} \otimes_{\mathcal{D}_{\tilde{Y}}} \tilde{\imath}^{*} \mathcal{D}_{\widetilde{X}} \otimes_{\tilde{\imath}^{-1}} \mathcal{O}_{\widetilde{X}} \mathcal{V}^{\prime}\right) & \simeq \mathcal{O}(\widetilde{Y}) \otimes_{\Gamma\left(\widetilde{Y}, \mathcal{D}_{\tilde{Y}}\right)} \Gamma\left(\widetilde{Y}, \tilde{\imath}^{*} \mathcal{D}_{\widetilde{X}} \otimes_{\tilde{\imath}^{-1}} \mathcal{O}_{\widetilde{X}} \mathcal{V}^{\prime}\right) \\
& \simeq \Gamma\left(\widetilde{Y}, \tilde{\imath}^{*} \mathcal{D}_{\widetilde{X}} \otimes_{\tilde{\imath}^{-1}} \mathcal{O}_{\widetilde{X}} \mathcal{V}^{\prime}\right) / \omega(\mathfrak{k}) \Gamma\left(\widetilde{Y}, \tilde{\imath}^{*} \mathcal{D}_{\widetilde{X}} \otimes_{\tilde{\imath}^{-1}} \mathcal{O}_{\widetilde{X}} \mathcal{V}^{\prime}\right)
\end{aligned}
$$

Let $e \in K$ be the identity element. Write $o:=e L \in \widetilde{Y}$ for the base point and $i_{o}:\{o\} \rightarrow \widetilde{Y}$ for the inclusion map. Let $\mathcal{I}_{o}$ be the maximal ideal of $\mathcal{O}_{\widetilde{Y}}$ corresponding to $o$. The fiber of $\tilde{\imath}^{*} \mathcal{D}_{\widetilde{X}} \otimes_{\tilde{\imath}^{-1} \mathcal{O}_{\widetilde{X}}} \mathcal{V}^{\prime}$ at $o$ is given by

$$
\begin{aligned}
W:=i_{o}^{*}\left(\tilde{\imath}^{*} \mathcal{D}_{\widetilde{X}} \otimes_{\tilde{\imath}^{-1}} \mathcal{O}_{\widetilde{X}} \mathcal{V}^{\prime}\right) \\
\\
\simeq \Gamma\left(\widetilde{Y}, \tilde{\imath}^{*} \mathcal{D}_{\widetilde{X}} \otimes_{\tilde{\imath}^{-1}} \mathcal{O}_{\widetilde{X}} \mathcal{V}^{\prime}\right) / \mathcal{I}_{o}(\widetilde{Y}) \Gamma\left(\widetilde{Y}, \tilde{\imath}^{*} \mathcal{D}_{\widetilde{X}} \otimes_{\tilde{\imath}^{-1} \mathcal{O}_{\widetilde{X}}} \mathcal{V}^{\prime}\right) .
\end{aligned}
$$

The actions $\rho$ and $\nu$ on $\tilde{\imath}^{*} \mathcal{D}_{\tilde{X}} \otimes_{\tilde{\imath}^{-1} \mathcal{O}_{\widetilde{X}}} \mathcal{V}^{\prime}$ induce a $\mathfrak{g}$-action and an $L$-action on $W$. With these actions, $W$ becomes a $(\mathfrak{g}, L)$-module and there is an isomorphism

$$
\varphi: U(\mathfrak{g}) \otimes_{U(\mathfrak{l})} V^{\prime} \stackrel{\sim}{\rightarrow} W .
$$

This can be proved by using [Osh11, Lemma 3.3] and Definition 3.3 (see the proof of [Osh11, Lemma 3.4]). Hence we have

$$
\Gamma\left(\widetilde{Y}, \tilde{\imath}^{*} \mathcal{D}_{\tilde{X}} \otimes_{\tilde{\imath}^{-1}} \mathcal{O}_{\widetilde{X}} \mathcal{V}^{\prime}\right) \simeq R(K) \otimes_{R(L)}\left(U(\mathfrak{g}) \otimes_{U(\mathfrak{l})} V^{\prime}\right) .
$$

The rest is the same as in [Osh11, Lemma 3.4]. 
Returning to the proof of Theorem 4.1, let us compute the cohomological induction $\left(P_{\mathfrak{h}, L}^{\mathfrak{g}, K}\right)_{s}\left(V \otimes \bigwedge^{\text {top }}(\mathfrak{g} / \mathfrak{h})\right)$ by using the standard resolution ([KV95, $\S I I .7])$. The standard resolution is a projective resolution of the $(\mathfrak{h}, L)$-module $V \otimes \bigwedge^{\text {top }}(\mathfrak{g} / \mathfrak{h})$ given by the complex

$$
U(\mathfrak{h}) \otimes_{U(\mathfrak{l})}\left(\bigwedge^{\bullet}(\mathfrak{h} / \mathfrak{l}) \otimes V \otimes \bigwedge^{\text {top }}(\mathfrak{g} / \mathfrak{h})\right)
$$

where the boundary map

$\partial^{\prime}: U(\mathfrak{h}) \otimes_{U(\mathfrak{l})}\left(\bigwedge^{d}(\mathfrak{h} / \mathfrak{l}) \otimes V \otimes \bigwedge^{\text {top }}(\mathfrak{g} / \mathfrak{h})\right) \rightarrow U(\mathfrak{h}) \otimes_{U(\mathfrak{l})}\left(\bigwedge^{d-1}(\mathfrak{h} / \mathfrak{l}) \otimes V \otimes \bigwedge^{\text {top }}(\mathfrak{g} / \mathfrak{h})\right)$

is

$$
\begin{aligned}
& D \otimes \overline{\xi_{1}} \wedge \cdots \wedge \overline{\xi_{d}} \otimes v \\
& \mapsto \sum_{i=1}^{d}(-1)^{i+1}\left(D \xi_{i} \otimes \overline{\xi_{1}} \wedge \cdots \wedge \widehat{\overline{\xi_{i}}} \wedge \cdots \wedge \overline{\xi_{d}} \otimes v-D \otimes \overline{\xi_{1}} \wedge \cdots \wedge \widehat{\overline{\xi_{i}}} \wedge \cdots \wedge \overline{\xi_{d}} \otimes \xi_{i} v\right) \\
& \quad+\sum_{1 \leq i<j \leq d}(-1)^{i+j} D \otimes \overline{\left[\xi_{i}, \xi_{j}\right]} \wedge \overline{\xi_{1}} \wedge \cdots \wedge \widehat{\overline{\xi_{i}}} \wedge \cdots \wedge \widehat{\overline{\xi_{j}}} \wedge \cdots \wedge \overline{\xi_{d}} \otimes v
\end{aligned}
$$

for $D \in U(\mathfrak{h}), \xi_{1}, \ldots, \xi_{d} \in \mathfrak{h}$, and $v \in V \otimes \bigwedge^{\text {top }}(\mathfrak{g} / \mathfrak{h})$. Therefore,

$$
\begin{aligned}
\left(P_{\mathfrak{h}, L}^{\mathfrak{g}, K}\right)_{u-s}(V & \left.\otimes \bigwedge^{\mathrm{top}}(\mathfrak{g} / \mathfrak{h})\right) \\
& \simeq \mathrm{H}^{s-u} P_{\mathfrak{h}, L}^{\mathfrak{g}, K}\left(U(\mathfrak{h}) \otimes_{U(\mathfrak{l})}\left(\bigwedge^{\bullet}(\mathfrak{h} / \mathfrak{l}) \otimes V \otimes \bigwedge^{\mathrm{top}}(\mathfrak{g} / \mathfrak{h})\right)\right) \\
& \simeq \mathrm{H}^{s-u} R(\mathfrak{g}, K) \otimes_{R(L)}\left(\bigwedge^{\bullet}(\mathfrak{h} / \mathfrak{l}) \otimes V \otimes \bigwedge^{\mathrm{top}}(\mathfrak{g} / \mathfrak{h})\right)
\end{aligned}
$$

where the boundary map

$$
\begin{aligned}
\partial^{\prime}: R(\mathfrak{g}, K) \otimes_{R(L)}\left(\bigwedge^{d}(\mathfrak{h} / \mathfrak{l})\right. & \left.\otimes V \otimes \bigwedge^{\mathrm{top}}(\mathfrak{g} / \mathfrak{h})\right) \\
& \rightarrow R(\mathfrak{g}, K) \otimes_{R(L)}\left(\bigwedge^{d-1}(\mathfrak{h} / \mathfrak{l}) \otimes V \otimes \bigwedge^{\mathrm{top}}(\mathfrak{g} / \mathfrak{h})\right)
\end{aligned}
$$

is given by

$$
\begin{aligned}
& D \otimes \overline{\xi_{1}} \wedge \cdots \wedge \overline{\xi_{d}} \otimes v \\
& \mapsto \sum_{i=1}^{d}(-1)^{i+1}\left(D \xi_{i} \otimes \overline{\xi_{1}} \wedge \cdots \wedge \widehat{\overline{\xi_{i}}} \wedge \cdots \wedge \overline{\xi_{d}} \otimes v-D \otimes \overline{\xi_{1}} \wedge \cdots \wedge \widehat{\overline{\xi_{i}}} \wedge \cdots \wedge \overline{\xi_{d}} \otimes \xi_{i} v\right) \\
& \quad+\sum_{1 \leq i<j \leq d}(-1)^{i+j} D \otimes \overline{\left[\xi_{i}, \xi_{j}\right]} \wedge \overline{\xi_{1}} \wedge \cdots \wedge \widehat{\overline{\xi_{i}}} \wedge \cdots \wedge \widehat{\overline{\xi_{j}}} \wedge \cdots \wedge \overline{\xi_{d}} \otimes v
\end{aligned}
$$

for $D \in R(\mathfrak{g}, K), \xi_{1}, \ldots, \xi_{d} \in \mathfrak{h}$, and $v \in V \otimes \bigwedge^{\text {top }}(\mathfrak{g} / \mathfrak{h})$.

Put

$$
V^{-d}:=\bigwedge^{d}(\mathfrak{h} / \mathfrak{l}) \otimes V \otimes \bigwedge^{\text {top }}(\mathfrak{g} / \mathfrak{h})
$$


for simplicity. We identify the fiber of $\mathcal{T}_{\widetilde{X} / X}$ with $\mathfrak{h} / \mathfrak{l}$ in the following way: if a vector field $\widetilde{\xi} \in \mathcal{T}_{\widetilde{X} / X}$ equals $-\xi_{\widetilde{X}}$ at the base point $e L \in \widetilde{X}$ for $\xi \in \mathfrak{h}$, then $\widetilde{\xi}$ takes the value $\bar{\xi} \in \mathfrak{h} / \mathfrak{l}$ at $e \in G$. Similarly, the fiber of $\Omega_{\widetilde{X} / X}^{\vee}$ is identified with $\bigwedge^{\text {top }}(\mathfrak{g} / \mathfrak{h})$. Then $\mathcal{V}^{-d}$ is associated with $V^{-d}$ by Examples 3.5 and 3.6. From (4.6) and (4.8) it is enough to show that the isomorphisms $\varphi$ given in Lemma 4.3 for $V^{\prime}=V^{-d}, 0 \leq d \leq \operatorname{dim}(\mathfrak{h} / \mathfrak{l})$, commute with the boundary maps, that is, the diagram

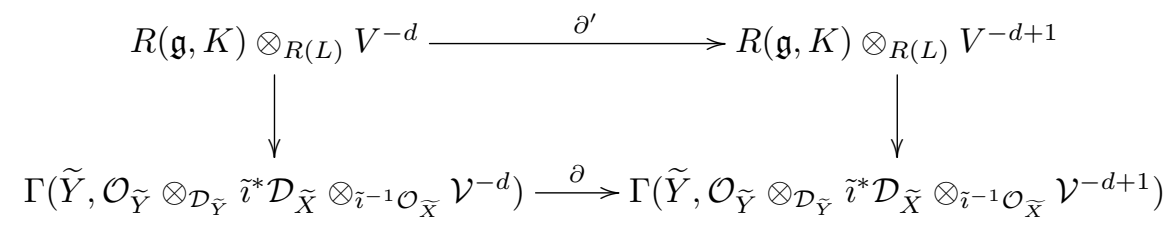

commutes. In view of the proof of Lemma 4.3, the above diagram is obtained by applying the functor $P_{\mathfrak{g}, L}^{\mathfrak{g}, K}$ to

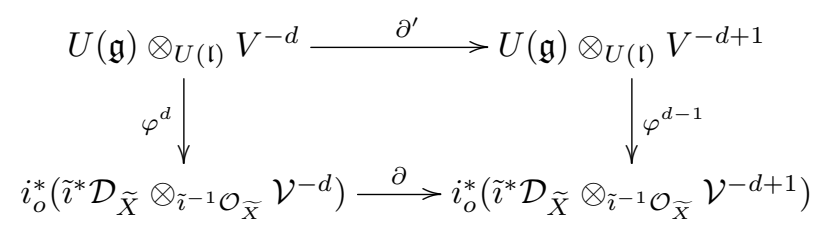

where $\varphi^{d}$ is the map $\varphi$ of (4.7) for $V^{\prime}=V^{-d}$. Therefore, it suffices to show that the diagram (4.9) commutes.

To see this, we use the following notation. A section $f \in \tilde{\imath}^{*} \mathcal{D}_{\widetilde{X}} \otimes_{\tilde{\imath}^{-1} \mathcal{O}_{\widetilde{X}}} \mathcal{V}^{-d}$ defines a section of $i_{o}^{*}\left(\tilde{\imath}^{*} \mathcal{D}_{\tilde{X}} \otimes_{\tilde{\imath}^{-1} \mathcal{O}_{\widetilde{X}}} \mathcal{V}^{-d}\right)$ and hence defines an element of $U(\mathfrak{g}) \otimes_{U(\mathfrak{l})}$ $V^{-d}$ via the isomorphism $\varphi^{d}$. We write $i_{o}^{*} f \in U(\mathfrak{g}) \otimes_{U(\mathfrak{l})} V^{-d}$ for this element. Put $Z:=H / L$ and write $i_{Z}: Z \rightarrow \widetilde{X}$ for the inclusion map. Then $i_{Z}(Z)=\pi^{-1}(\{o\})$ and there is a canonical isomorphism $i_{Z}^{*} \mathcal{T}_{\widetilde{X} / X} \simeq \mathcal{T}_{Z}$. For $\xi_{1}, \ldots, \xi_{d} \in \mathfrak{h}$ and $v \in$ $V \otimes \bigwedge^{\text {top }}(\mathfrak{g} / \mathfrak{h})$, put

$$
m:=\overline{\xi_{1}} \wedge \cdots \wedge \overline{\xi_{d}} \otimes v \in V^{-d} .
$$

We will choose sections $\widetilde{\xi}_{i} \in \tilde{\imath}^{-1} \mathcal{T}_{\widetilde{X} / X}$ and $\widetilde{v} \in \pi_{K}^{-1}\left(\mathcal{V} \otimes_{i^{-1} \mathcal{O}_{X}} i^{-1} \Omega_{X}^{\vee}\right)$ on a neighborhood of the base point $o \in \tilde{Y}$ in the following way. Take $\widetilde{\xi}_{i} \in \mathcal{T}_{\widetilde{X} / X}$ such that $\left.\widetilde{\xi}_{i}\right|_{Z} \in i_{Z}^{*} \mathcal{T}_{\widetilde{X} / X}$ corresponds to $-\left(\xi_{i}\right)_{Z}$. It gives a section of $\tilde{\imath}^{-1} \mathcal{T}_{\widetilde{X} / X}$, which we denote by the same letter $\widetilde{\xi}_{i}$. We take a section $\widetilde{v} \in \pi_{K}^{-1}\left(\mathcal{V} \otimes_{i^{-1} \mathcal{O}_{X}} i^{-1} \Omega_{X}^{\vee}\right)$ on a neighborhood of $o$ such that $i_{o}^{*} \widetilde{v}$ corresponds to $v$. Define a section $\widetilde{m} \in \mathcal{V}^{-d}$ in a neighborhood of $o$ as

$$
\widetilde{m}:=\widetilde{\xi}_{1} \wedge \cdots \wedge \widetilde{\xi}_{d} \otimes \widetilde{v} \in \mathcal{V}^{-d}
$$


Then the element $\varphi^{d}(1 \otimes m)$ is represented by the section

$$
1 \otimes \widetilde{m} \in \tilde{\imath}^{*} \mathcal{D}_{\widetilde{X}} \otimes_{\tilde{\imath}^{-1} \mathcal{O}_{\widetilde{X}}} \mathcal{V}^{-d}
$$

in other words, $i_{o}^{*}(1 \otimes \widetilde{m})=1 \otimes m$.

We have

$$
\begin{aligned}
\partial(1 \otimes \widetilde{m})= & \sum_{i=1}^{d}(-1)^{i+1}\left(\widetilde{\xi}_{i} \otimes \widetilde{\xi}_{1} \wedge \cdots \wedge \widehat{\widetilde{\xi}}_{i} \wedge \cdots \wedge \widetilde{\xi}_{d} \otimes \widetilde{v}\right) \\
& +\sum_{1 \leq i<j \leq d}(-1)^{i+j}\left(1 \otimes\left[\widetilde{\xi}_{i}, \widetilde{\xi}_{j}\right] \wedge \widetilde{\xi}_{1} \wedge \cdots \wedge \widetilde{\widetilde{\xi}}_{i} \wedge \cdots \wedge \widetilde{\widetilde{\xi}}_{j} \wedge \cdots \wedge \widetilde{\xi}_{d} \otimes \widetilde{v}\right)
\end{aligned}
$$

and

$$
\begin{aligned}
& \partial^{\prime}(1 \otimes m) \\
& =\sum_{i=1}^{d}(-1)^{i+1}\left(\xi_{i} \otimes \overline{\xi_{1}} \wedge \cdots \wedge \widehat{\overline{\xi_{i}}} \wedge \cdots \wedge \overline{\xi_{d}} \otimes v-1 \otimes \overline{\xi_{1}} \wedge \cdots \wedge \widehat{\overline{\xi_{i}}} \wedge \cdots \wedge \overline{\xi_{d}} \otimes \xi_{i} v\right) \\
& \quad+\sum_{1 \leq i<j \leq d}(-1)^{i+j}\left(1 \otimes \overline{\left[\xi_{i}, \xi_{j}\right]} \wedge \overline{\xi_{1}} \wedge \cdots \wedge \widehat{\overline{\xi_{i}}} \wedge \cdots \wedge \widehat{\overline{\xi_{j}}} \wedge \cdots \wedge \overline{\xi_{d}} \otimes v\right) .
\end{aligned}
$$

Since $\left.\widetilde{\xi}_{i}\right|_{Z}$ corresponds to $-\left(\xi_{i}\right)_{Z}$, the vector fields $\widetilde{\xi}_{i}$ and $\left(\xi_{i}\right)_{\widetilde{X}}$ satisfy the relation $\widetilde{\xi}_{i}=-\left(\xi_{i}\right)_{\widetilde{X}}$ at $o$. Recall that the $\mathfrak{g}$-action on $\mathcal{T}_{\widetilde{X} / X}$ is defined as the differential of the $G$-equivariant structure on it. Hence our choice implies that $\left.\xi_{i} \cdot \widetilde{\xi}_{j}\right|_{Z}=$ $-\left(\left[\xi_{i}, \xi_{j}\right]\right)_{Z}$. As a result,

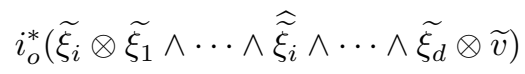

$$
\begin{aligned}
& =i_{o}^{*}\left(\rho\left(\xi_{i}\right)\left(1 \otimes \widetilde{\xi}_{1} \wedge \cdots \wedge \widehat{\widetilde{\xi}}_{i} \wedge \cdots \wedge \widetilde{\xi}_{d} \otimes \widetilde{v}\right)\right)-i_{o}^{*}\left(1 \otimes \widetilde{\xi}_{1} \wedge \cdots \wedge \widehat{\widetilde{\xi}}_{i} \wedge \cdots \wedge \widetilde{\xi}_{d} \otimes \xi_{i} \widetilde{v}\right) \\
& -\sum_{1 \leq i<j \leq d} i_{o}^{*}\left(1 \otimes \widetilde{\xi}_{1} \wedge \cdots \wedge \widehat{\widetilde{\xi}}_{i} \wedge \cdots \wedge \widetilde{\xi}_{j-1} \wedge\left(\xi_{i} \cdot \widetilde{\xi}_{j}\right) \wedge \widetilde{\xi}_{j+1} \wedge \cdots \wedge \widetilde{\xi}_{d} \otimes \widetilde{v}\right) \\
& -\sum_{1 \leq j<i \leq d} i_{o}^{*}\left(1 \otimes \widetilde{\xi}_{1} \wedge \cdots \wedge \widetilde{\xi}_{j-1} \wedge\left(\xi_{i} \cdot \widetilde{\xi}_{j}\right) \wedge \widetilde{\xi}_{j+1} \wedge \cdots \wedge \widehat{\widetilde{\xi}}_{i} \wedge \cdots \wedge \widetilde{\xi}_{d} \otimes \widetilde{v}\right) \\
& =\xi_{i} \otimes \overline{\xi_{1}} \wedge \cdots \wedge \widehat{\overline{\xi_{i}}} \wedge \cdots \wedge \overline{\xi_{d}} \otimes v-1 \otimes \overline{\xi_{1}} \wedge \cdots \wedge \widehat{\overline{\xi_{i}}} \wedge \cdots \wedge \overline{\overline{\xi_{d}}} \otimes \xi_{i} v \\
& +\sum_{1 \leq i<j \leq d}(-1)^{j+1}\left(1 \otimes \overline{\left[\xi_{i}, \xi_{j}\right]} \wedge \overline{\xi_{1}} \wedge \cdots \wedge \widehat{\overline{\xi_{i}}} \wedge \cdots \wedge \widehat{\overline{\xi_{j}}} \wedge \cdots \wedge \overline{\xi_{d}} \otimes v\right) \\
& +\sum_{1 \leq j<i \leq d}(-1)^{j}\left(1 \otimes \overline{\left[\xi_{i}, \xi_{j}\right]} \wedge \overline{\xi_{1}} \wedge \cdots \wedge \widehat{\overline{\xi_{j}}} \wedge \cdots \wedge \widehat{\overline{\xi_{i}}} \wedge \cdots \wedge \overline{\xi_{d}} \otimes v\right)
\end{aligned}
$$


Moreover, $\left.\left[\widetilde{\xi}_{i}, \widetilde{\xi}_{j}\right]\right|_{Z}$ corresponds to $\left[-\left(\xi_{i}\right)_{Z},-\left(\xi_{j}\right)_{Z}\right]=\left(\left[\xi_{i}, \xi_{j}\right]\right)_{Z}$. Hence

$$
\begin{aligned}
i_{o}^{*}\left(1 \otimes\left[\widetilde{\xi}_{i}, \widetilde{\xi}_{j}\right] \wedge \widetilde{\xi}_{1}\right. & \left.\wedge \wedge \widehat{\widetilde{\xi}}_{i} \wedge \cdots \wedge \widehat{\widetilde{\xi}}_{j} \wedge \cdots \wedge{\widetilde{\xi_{d}}} \otimes \widetilde{v}\right) \\
& =-1 \otimes \overline{\left[\xi_{i}, \xi_{j}\right]} \wedge \overline{\xi_{1}} \wedge \cdots \wedge \widehat{\overline{\xi_{i}}} \wedge \cdots \wedge \widehat{\overline{\xi_{j}}} \wedge \cdots \wedge \overline{\xi_{d}} \otimes v .
\end{aligned}
$$

We thus conclude that

$$
\begin{aligned}
& \left(\varphi^{d-1}\right)^{-1} \circ \partial \circ \varphi^{d}(1 \otimes m)=i_{o}^{*}(\partial(1 \otimes \widetilde{m})) \\
& =i_{o}^{*}\left(\sum_{i=1}^{d}(-1)^{i+1}\left(\widetilde{\xi}_{i} \otimes \widetilde{\xi}_{1} \wedge \cdots \wedge \widehat{\widetilde{\xi}}_{i} \wedge \cdots \wedge \widetilde{\xi}_{d} \otimes \widetilde{v}\right)\right. \\
& \left.+\sum_{1 \leq i<j \leq d}(-1)^{i+j}\left(1 \otimes\left[\widetilde{\xi}_{i}, \widetilde{\xi}_{j}\right] \wedge \widetilde{\xi}_{1} \wedge \cdots \wedge \widehat{\widetilde{\xi}}_{i} \wedge \cdots \wedge \widehat{\widetilde{\xi}}_{j} \wedge \cdots \wedge \widetilde{\xi}_{d} \otimes \widetilde{v}\right)\right) \\
& =\sum_{i=1}^{d}(-1)^{i+1}\left(\xi_{i} \otimes \overline{\xi_{1}} \wedge \cdots \wedge \widehat{\overline{\xi_{i}}} \wedge \cdots \wedge \overline{\xi_{d}} \otimes v-1 \otimes \overline{\xi_{1}} \wedge \cdots \wedge \widehat{\overline{\xi_{i}}} \wedge \cdots \wedge \overline{\xi_{d}} \otimes \xi_{i} v\right. \\
& +\sum_{1 \leq i<j \leq d}(-1)^{j+1}\left(1 \otimes \overline{\left[\xi_{i}, \xi_{j}\right]} \wedge \overline{\xi_{1}} \wedge \cdots \wedge \widehat{\overline{\xi_{i}}} \wedge \cdots \wedge \widehat{\overline{\xi_{j}}} \wedge \cdots \wedge \overline{\xi_{d}} \otimes v\right) \\
& \left.+\sum_{1 \leq j<i \leq d}(-1)^{j}\left(1 \otimes \overline{\left[\xi_{i}, \xi_{j}\right]} \wedge \overline{\xi_{1}} \wedge \cdots \wedge \widehat{\widehat{\xi_{j}}} \wedge \cdots \wedge \widehat{\widehat{\xi_{i}}} \wedge \cdots \wedge \overline{\xi_{d}} \otimes v\right)\right) \\
& +\sum_{1 \leq i<j \leq d}(-1)^{i+j+1}\left(1 \otimes \overline{\left[\xi_{i}, \xi_{j}\right]} \wedge \overline{\xi_{1}} \wedge \cdots \wedge \widehat{\widehat{\xi_{i}}} \wedge \cdots \wedge \widehat{\widehat{\xi_{j}}} \wedge \cdots \wedge \overline{\xi_{d}} \otimes v\right) \\
& =\sum_{i=1}^{d}(-1)^{i+1}\left(\xi_{i} \otimes \overline{\xi_{1}} \wedge \cdots \wedge \widehat{\overline{\xi_{i}}} \wedge \cdots \wedge \overline{\xi_{d}} \otimes v-1 \otimes \overline{\xi_{1}} \wedge \cdots \wedge \widehat{\overline{\xi_{i}}} \wedge \cdots \wedge \overline{\xi_{d}} \otimes \xi_{i} v\right) \\
& +\sum_{1 \leq i<j \leq d}(-1)^{i+j}\left(1 \otimes \overline{\left[\xi_{i}, \xi_{j}\right]} \wedge \overline{\xi_{1}} \wedge \cdots \wedge \widehat{\overline{\xi_{i}}} \wedge \cdots \wedge \widehat{\overline{\xi_{j}}} \wedge \cdots \wedge \overline{\xi_{d}} \otimes v\right) \\
& =\partial^{\prime}(1 \otimes m) \text {. }
\end{aligned}
$$

Since $\partial, \partial^{\prime}$ and $\varphi^{d}$ commute with $\mathfrak{g}$-actions,

$$
\partial\left(\varphi^{d}(D \otimes m)\right)=D \partial\left(\varphi^{d}(1 \otimes m)\right)=D \varphi^{d-1}\left(\partial^{\prime}(1 \otimes m)\right)=\varphi^{d-1}\left(\partial^{\prime}(D \otimes m)\right)
$$

for $D \in U(\mathfrak{g})$. Consequently, the diagram (4.9) commutes and the proof of the theorem is complete.

\section{$\S 5$. Construction of modules}

In this section, we will construct an $i^{-1} \widetilde{\mathfrak{g}}_{X}$-module $\mathcal{V}$ associated with an $(\mathfrak{h}, M)$ module $V$, which can be used in Section 4 for the realization of cohomologically induced modules. 
Let $\mathcal{V}_{Y}$ be the $K$-equivariant quasi-coherent $\mathcal{O}_{Y}$-module with typical fiber the $M$-module $V$. Let $p: \mathcal{O}_{X} \otimes_{\mathbb{C}} \mathfrak{g} \rightarrow \mathcal{T}_{X}$ be the map given by $f \otimes \xi \mapsto f \xi_{X}$ and put $\mathcal{H}:=\operatorname{ker} p$. The $\mathcal{O}_{X}$-module $\mathcal{H}$ is $G$-equivariant with typical fiber $\mathfrak{h}$. Hence a section $\xi \in \mathcal{H}$ is identified with an $\mathfrak{h}$-valued regular function on a subset of $G$ satisfying $\xi(g h)=\operatorname{Ad}\left(h^{-1}\right)(\xi(g))$ for $h \in H$. Let $\xi, \xi^{\prime} \in \mathcal{H}$. By regarding $\tilde{\mathfrak{g}}_{X}=$ $\mathcal{O}_{X} \otimes_{\mathbb{C}} \mathfrak{g}$ as a submodule of $U\left(\widetilde{\mathfrak{g}}_{X}\right)=\mathcal{O}_{X} \otimes_{\mathbb{C}} U(\mathfrak{g})$, we have $\left[\xi, \xi^{\prime}\right]:=\xi \xi^{\prime}-\xi^{\prime} \xi \in \mathcal{H}$ and $\left[\xi, \xi^{\prime}\right](g)=\left[\xi(g), \xi^{\prime}(g)\right]$ with the identification above. If we write $\xi=\sum_{i} f_{i} \otimes \xi_{i}$ for $f_{i} \in \mathcal{O}_{X}$ and $\xi_{i} \in \mathfrak{g}$, then $\xi(g)=\sum_{i} f_{i}(g) \operatorname{Ad}\left(g^{-1}\right)\left(\xi_{i}\right)$.

Let $\mathcal{A}$ be the subalgebra of $i^{-1} U\left(\widetilde{\mathfrak{g}}_{X}\right)=i^{-1} \mathcal{O}_{X} \otimes U(\mathfrak{g})$ generated by $i^{-1} \mathcal{H}, 1 \otimes \mathfrak{k}$, and $i^{-1} \mathcal{O}_{X} \otimes 1$. We view $i^{-1} U\left(\widetilde{\mathfrak{g}}_{X}\right)$ as an $i^{-1} \mathcal{O}_{X}$-module and consider the inverse image $\mathcal{O}_{Y} \otimes_{i^{-1} \mathcal{O}_{X}} i^{-1} U\left(\widetilde{\mathfrak{g}}_{X}\right)\left(\simeq \mathcal{O}_{Y} \otimes U(\mathfrak{g})\right)$ of $U\left(\widetilde{\mathfrak{g}}_{X}\right)$. Let $\overline{\mathcal{A}}$ be the image of the $\operatorname{map} \mathcal{O}_{Y} \otimes_{i^{-1} \mathcal{O}_{X}} \mathcal{A} \rightarrow \mathcal{O}_{Y} \otimes_{i^{-1} \mathcal{O}_{X}} i^{-1} U\left(\widetilde{\mathfrak{g}}_{X}\right)$ so that $\overline{\mathcal{A}} \simeq \mathcal{A} /\left(\mathcal{A} \cap\left(i^{-1} \mathcal{I}_{Y} \otimes U(\mathfrak{g})\right)\right)$. Since $\mathcal{A} \cdot\left(i^{-1} \mathcal{I}_{Y} \otimes U(\mathfrak{g})\right) \subset i^{-1} \mathcal{I}_{Y} \otimes U(\mathfrak{g})$ in the algebra $i^{-1} U\left(\widetilde{\mathfrak{g}}_{X}\right)$, the algebra structure of $\mathcal{A}$ induces that of $\overline{\mathcal{A}}$, and $\mathcal{O}_{Y} \otimes_{i^{-1} \mathcal{O}_{X}} i^{-1} U\left(\widetilde{\mathfrak{g}}_{X}\right)$ becomes a left $\overline{\mathcal{A}}$ module.

We give a left $\overline{\mathcal{A}}$-module structure on $\mathcal{V}_{Y}$ in the following way. We view a local section of $\mathcal{V}_{Y}$ as a $V$-valued regular function on a subset of $K$ and define a $\left(1 \otimes i^{-1} \mathcal{H}\right)$-action and an $\left(\mathcal{O}_{Y} \otimes 1\right)$-action by

$$
((1 \otimes \xi) v)(k)=\xi(i(k)) v(k), \quad(f \otimes 1) v=f v
$$

for $\xi \in i^{-1} \mathcal{H}, v \in \mathcal{V}_{Y}, f \in \mathcal{O}_{Y}$, and $k \in K$; define a $(1 \otimes \mathfrak{k})$-action on $\mathcal{V}_{Y}$ by differentiating the $K$-action on $\mathcal{V}_{Y}$. These actions are compatible in the following sense: if $f_{i} \in i^{-1} \mathcal{O}_{X}, \eta_{i} \in \mathfrak{k}$ and $\xi \in i^{-1} \mathcal{H}$ satisfy

$$
\sum_{i}\left(f_{i} \otimes \eta_{i}\right)-\xi \in i^{-1} \mathcal{I}_{Y} \otimes \mathfrak{g}
$$

then we have

$$
\sum_{i}\left(\left.f_{i}\right|_{Y} \otimes 1\right)\left(\left(1 \otimes \eta_{i}\right) v\right)=(1 \otimes \xi) v
$$

for $v \in \mathcal{V}_{Y}$. In the proposition below, we will see that these actions give a welldefined $\overline{\mathcal{A}}$-module structure.

Let $\mathcal{V}:=\mathcal{H}^{\circ} m_{\overline{\mathcal{A}}}\left(\mathcal{O}_{Y} \otimes_{i^{-1} \mathcal{O}_{X}} i^{-1} U\left(\widetilde{\mathfrak{g}}_{X}\right), \mathcal{V}_{Y}\right)$, namely, $\mathcal{V}$ consists of the sections $v \in \mathcal{H o m}_{\mathbb{C}}\left(\mathcal{O}_{Y} \otimes_{i^{-1} \mathcal{O}_{X}} i^{-1} U\left(\widetilde{\mathfrak{g}}_{X}\right), \mathcal{V}_{Y}\right)$ satisfying

$$
\begin{aligned}
& v((1 \otimes \xi)(f \otimes D))=(1 \otimes \xi)(v(f \otimes D)), \\
& v((1 \otimes \eta)(f \otimes D))=(1 \otimes \eta)(v(f \otimes D)), \text { and } \\
& v\left(f^{\prime} f \otimes D\right)=\left(f^{\prime} \otimes 1\right)(v(f \otimes D))
\end{aligned}
$$


for $f, f^{\prime} \in \mathcal{O}_{Y}, D \in U(\mathfrak{g}), \eta \in \mathfrak{k}$, and $\xi \in i^{-1} \mathcal{H}$. We endow $\mathcal{V}$ with an $i^{-1} \widetilde{\mathfrak{g}}_{X}$-module structure by defining $(f \otimes D) \cdot v$ as

$$
((f \otimes D) \cdot v)\left(f^{\prime} \otimes D^{\prime}\right)=v\left(f^{\prime} \otimes\left(1 \otimes D^{\prime}\right)(f \otimes D)\right)
$$

for $v \in \mathcal{V}, f \in i^{-1} \mathcal{O}_{X}, f^{\prime} \in \mathcal{O}_{Y}$, and $D, D^{\prime} \in U(\mathfrak{g})$.

Proposition 5.1. Let $V$ be an $(\mathfrak{h}, M)$-module. Then the left $\overline{\mathcal{A}}$-action on $\mathcal{V}_{Y}$ given above is well-defined, and the $i^{-1} \widetilde{\mathfrak{g}}_{X}$-module

$$
\mathcal{V}:=\mathcal{H o m}_{\overline{\mathcal{A}}}\left(\mathcal{O}_{Y} \otimes_{i^{-1} \mathcal{O}_{X}} U\left(\widetilde{\mathfrak{g}}_{X}\right), \mathcal{V}_{Y}\right)
$$

is associated with $V$ in the sense of Definition 3.3.

Proof. Let $k_{0} \in K$ and $y_{0}:=k_{0} M \in Y$. We fix a trivialization near $y_{0}$ in the following way. Take sections $\xi_{1}, \ldots, \xi_{n} \in i^{-1} \mathcal{H}$ on a neighborhood $U$ of $y_{0}$ in $Y$ such that the map

$$
\left.\left.\left(i^{-1} \mathcal{O}_{X}\right)^{\oplus n}\right|_{U} \rightarrow\left(i^{-1} \mathcal{H}\right)\right|_{U}, \quad\left(f_{1}, \ldots, f_{n}\right) \mapsto \sum_{i=1}^{n} f_{i} \xi_{i},
$$

is an isomorphism. Take elements $\eta_{1}, \ldots, \eta_{m} \in \mathfrak{k}$ that form a basis of the quotient space $\mathfrak{k} / \operatorname{Ad}\left(k_{0}\right)(\mathfrak{m})$ and take $\zeta_{1}, \ldots, \zeta_{l} \in \mathfrak{g}$ such that $\eta_{1}, \ldots, \eta_{m}, \zeta_{1}, \ldots, \zeta_{l}$ form a basis of the quotient space $\mathfrak{g} / \operatorname{Ad}\left(i\left(k_{0}\right)\right) \mathfrak{h}$. Modifying $U$ if necessary, we get an isomorphism

$$
\begin{aligned}
\left.\left(i^{-1} \mathcal{O}_{X}\right)^{\oplus n+m+l}\right|_{U} & \left.\rightarrow\left(i^{-1} \mathcal{O}_{X} \otimes_{\mathbb{C}} \mathfrak{g}\right)\right|_{U}, \\
\left(f_{1}, \ldots, f_{n}, g_{1}, \ldots, g_{m}, h_{1}, \ldots, h_{l}\right) & \mapsto \sum_{i=1}^{n} f_{i} \xi_{i}+\sum_{i=1}^{m}\left(g_{i} \otimes \eta_{i}\right)+\sum_{i=1}^{l}\left(h_{i} \otimes \zeta_{i}\right) .
\end{aligned}
$$

For integers $s, t \geq 0$, let

$$
I_{s, t}:=\{\boldsymbol{i}=(i(1), \ldots, i(s)): 1 \leq i(1) \leq \cdots \leq i(s) \leq t\}, \quad I_{t}:=\coprod_{s=0}^{\infty} I_{s, t} .
$$

If $s=0$, the set $I_{0, t}$ consists of one element (). For $\boldsymbol{i}=(i(1), \ldots, i(s)) \in I_{s, l}$, we put $\zeta_{\boldsymbol{i}}:=1 \otimes \zeta_{i(1)} \cdots \zeta_{i(s)} \in i^{-1} \mathcal{O}_{X} \otimes U(\mathfrak{g})$. If $s=0$ and $\boldsymbol{i}=()$ then put $\zeta_{\boldsymbol{i}}:=1 \otimes 1$. In the same way, for $\boldsymbol{i}^{\prime}=\left(i^{\prime}(1), \ldots, i^{\prime}(s)\right) \in I_{s, n}$ and $\boldsymbol{i}^{\prime \prime}=\left(i^{\prime \prime}(1), \ldots, i^{\prime \prime}(s)\right) \in I_{s, m}$, put $\xi_{i^{\prime}}:=\xi_{i^{\prime}(1)} \cdots \xi_{i^{\prime}(s)}$ and $\eta_{i^{\prime \prime}}:=1 \otimes \eta_{i^{\prime \prime}(1)} \cdots \eta_{i^{\prime \prime}(s)}$. From the isomorphism (5.2) and the Poincaré-Birkhoff-Witt theorem, we see that a section of $\left.i^{-1} U\left(\widetilde{\mathfrak{g}}_{X}\right)\right|_{U}$ is uniquely written as

$$
\sum_{i \in I_{l}, \boldsymbol{i}^{\prime} \in I_{n}, \boldsymbol{i}^{\prime \prime} \in I_{m}} f_{\boldsymbol{i}, i^{\prime}, i^{\prime \prime}} \xi_{\boldsymbol{i}^{\prime}} \eta_{\boldsymbol{i}^{\prime \prime}} \zeta_{\boldsymbol{i}}
$$


where $f_{\boldsymbol{i}, \boldsymbol{i}^{\prime}, \boldsymbol{i}^{\prime \prime}} \in i^{-1} \mathcal{O}_{X}$, and $f_{\boldsymbol{i}, \boldsymbol{i}^{\prime}, \boldsymbol{i}^{\prime \prime}}=0$ except for finitely many $\left(\boldsymbol{i}, \boldsymbol{i}^{\prime}, \boldsymbol{i}^{\prime \prime}\right)$. Hence a section of $\left.\left(\mathcal{O}_{Y} \otimes_{i^{-1} \mathcal{O}_{X}} i^{-1} U\left(\widetilde{\mathfrak{g}}_{X}\right)\right)\right|_{U}$ is uniquely written as a finite sum $\sum_{i, i^{\prime}, i^{\prime \prime}} f_{i, i^{\prime}, i^{\prime \prime}} \xi_{i^{\prime}} \eta_{i^{\prime \prime}} \zeta_{i}$ for $f_{i, i^{\prime}, i^{\prime \prime}} \in \mathcal{O}_{Y}$.

Lemma 5.2. The subsheaf $\left.\overline{\mathcal{A}}\right|_{U}$ of $\mathcal{O}_{Y} \otimes_{i^{-1} \mathcal{O}_{X}} i^{-1} U\left(\widetilde{\mathfrak{g}}_{X}\right)$ consists of the sections written as a finite sum

$$
\sum_{i^{\prime} \in I_{n}, i^{\prime \prime} \in I_{m}} f_{i^{\prime}, i^{\prime \prime}} \otimes \xi_{i^{\prime}} \eta_{i^{\prime \prime}}
$$

for $f_{i^{\prime}, i^{\prime \prime}} \in \mathcal{O}_{Y}$.

Proof. It is enough to prove that for any section $\left.a \in \mathcal{A}\right|_{U}$ there exist functions $f_{\boldsymbol{i}^{\prime}, i^{\prime \prime}} \in i^{-1} \mathcal{O}_{X}$ such that

$$
a-\sum_{\boldsymbol{i}^{\prime}, \boldsymbol{i}^{\prime \prime}} f_{\boldsymbol{i}^{\prime}, \boldsymbol{i}^{\prime \prime}} \xi_{\boldsymbol{i}^{\prime}} \eta_{\boldsymbol{i}^{\prime \prime}} \in i^{-1} \mathcal{I}_{Y} \otimes U(\mathfrak{g}) .
$$

For this we consider relations in the algebra $i^{-1} U\left(\widetilde{\mathfrak{g}}_{X}\right)$. By our choice of $\xi_{1}, \ldots, \xi_{n}$ and $\eta_{1}, \ldots, \eta_{m}$, we can find $f_{i}, g_{i} \in i^{-1} \mathcal{O}_{X}$ for each $\eta \in \mathfrak{k}$ such that

$$
(1 \otimes \eta)-\left(\sum_{i=1}^{n} f_{i} \xi_{i}+\sum_{i=1}^{m} g_{i} \otimes \eta_{i}\right) \in i^{-1} \mathcal{I}_{Y} \otimes U(\mathfrak{g}) .
$$

We also have

$$
\left[\xi_{i}, f \otimes 1\right]=0, \quad\left[1 \otimes \eta, 1 \otimes \eta^{\prime}\right]=1 \otimes\left[\eta, \eta^{\prime}\right], \quad[1 \otimes \eta, f \otimes 1]=\left(\eta_{X}(f)\right) \otimes 1
$$

for $f \in i^{-1} \mathcal{O}_{X}, \eta, \eta^{\prime} \in \mathfrak{k}$. Further $\left[\xi_{i}, \xi_{j}\right],\left[1 \otimes \eta_{i}, \xi_{j}\right] \in i^{-1} \mathcal{H}$ and hence there exist $f_{i, j, k}, g_{i, j, k} \in i^{-1} \mathcal{O}_{X}$ such that

$$
\left[\xi_{i}, \xi_{j}\right]=\sum_{k=1}^{n} f_{i, j, k} \xi_{k}, \quad\left[1 \otimes \eta_{i}, \xi_{j}\right]=\sum_{k=1}^{n} g_{i, j, k} \xi_{k} .
$$

Since $\mathcal{A}$ is generated by $i^{-1} \mathcal{H}, 1 \otimes \mathfrak{k}$ and $i^{-1} \mathcal{O}_{X} \otimes 1$, we can prove (5.3) by using these relations iteratively and using $\mathcal{A}\left(i^{-1} \mathcal{I}_{Y} \otimes U(\mathfrak{g})\right) \subset i^{-1} \mathcal{I}_{Y} \otimes U(\mathfrak{g})$.

From the lemma above and its proof, we see that the algebra $\overline{\mathcal{A}}$ is generated by $\mathcal{O}_{Y} \otimes 1,1 \otimes \xi_{1}, \ldots, 1 \otimes \xi_{n}$, and $1 \otimes \mathfrak{k}$ with the relations:

$$
\begin{aligned}
& 1 \otimes \eta=\sum_{i=1}^{n} f_{i} \otimes \xi_{i}+\sum_{i=1}^{m} g_{i} \otimes \eta_{i}, \\
& {\left[1 \otimes \xi_{i}, f \otimes 1\right]=0, \quad\left[1 \otimes \eta, 1 \otimes \eta^{\prime}\right]=1 \otimes\left[\eta, \eta^{\prime}\right], \quad[1 \otimes \eta, f \otimes 1]=\left(\eta_{Y}(f)\right) \otimes 1,} \\
& {\left[1 \otimes \xi_{i}, 1 \otimes \xi_{j}\right]=\sum_{k=1}^{n} f_{i, j, k} \otimes \xi_{k}, \quad\left[1 \otimes \eta_{i}, 1 \otimes \xi_{j}\right]=\sum_{k=1}^{n} g_{i, j, k} \otimes \xi_{k},}
\end{aligned}
$$


where $f_{i}, g_{i}, f_{i, j, k}, g_{i, j, k}$ are the restrictions to $Y$ of the corresponding functions in the proof of Lemma 5.2 and $f \in \mathcal{O}_{Y}, \eta, \eta^{\prime} \in \mathfrak{k}$. We can check that these relations are compatible with the action on $\mathcal{V}_{Y}\left(\right.$ see $(5.1)$ ) and hence the $\overline{\mathcal{A}}$-action on $\mathcal{V}_{Y}$ is well-defined.

By Lemma $5.2,\left.\left(\mathcal{O}_{Y} \otimes_{i^{-1} \mathcal{O}_{X}} i^{-1} U\left(\widetilde{\mathfrak{g}}_{X}\right)\right)\right|_{U}$ is a free $\left.\overline{\mathcal{A}}\right|_{U^{-a l g e b r a}}$ with basis $1 \otimes \zeta_{\boldsymbol{i}}$. Therefore, the map

$$
\phi:\left.\left.\mathcal{V}\right|_{U} \rightarrow \prod_{i \in I_{l}} \mathcal{V}_{Y}\right|_{U}
$$

given by $\phi(v)=\left(v\left(1 \otimes \zeta_{\boldsymbol{i}}\right)\right)_{\boldsymbol{i}}$ is bijective.

Our choice of $\zeta_{1}, \ldots, \zeta_{l}$ implies that they form a basis of the normal tangent bundle of $U$ in $X$. Since $\phi$ is bijective, we see that

$$
\phi\left(\left.\left(i^{-1} \mathcal{I}_{Y}\right)^{p} \mathcal{V}\right|_{U}\right)=\left.\prod_{s=p}^{\infty} \prod_{i \in I_{s, l}} \mathcal{V}_{Y}\right|_{U}
$$

and hence

$$
\left.\left.\left(\mathcal{V} /\left(i^{-1} \mathcal{I}_{Y}\right)^{p} \mathcal{V}\right)\right|_{U} \simeq \prod_{s=0}^{p-1} \prod_{i \in I_{s, l}} \mathcal{V}_{Y}\right|_{U}
$$

If we endow the right side of the last isomorphism with an $\mathcal{O}_{Y_{p}}$-module structure via this isomorphism, it is written as follows. Let $f \in i^{-1} \mathcal{O}_{X}$ and $v=\left(v_{\boldsymbol{i}}\right)_{\boldsymbol{i}}$. For a subset $A \subset\{1, \ldots, s\}$ with $A=\{a(1), \ldots, a(t)\}, a(1)<\cdots<a(t)$, and for $\boldsymbol{i}=(i(1), \ldots, i(s)) \in I_{s, l}$, let $\{b(1), \ldots, b(s-t)\}=\{1, \ldots, s\} \backslash A$ with $b(1)<\cdots<$ $b(s-t)$ and put $\boldsymbol{i}^{\prime}:=(i(b(1)), \ldots, i(b(s-t))) \in I_{s-t, l}$. Then the $\boldsymbol{i}$-term of $f \cdot v$ is

$$
(f \cdot v)_{\boldsymbol{i}}=\left.\sum_{A \subset\{1, \ldots, s\}}\left(\left(\zeta_{i(a(1))}\right)_{X} \cdots\left(\zeta_{i(a(t))}\right)_{X} f\right)\right|_{U} \cdot v_{\boldsymbol{i}^{\prime}}
$$

On the right side here, we use the $\mathcal{O}_{Y}$-action on $\mathcal{V}_{Y}$. This $i^{-1} \mathcal{O}_{X}$-action on $\left.\prod_{s=0}^{p-1} \prod_{i \in I_{s, l}} \mathcal{V}_{Y}\right|_{U}$ induces an $\mathcal{O}_{Y_{p}}$-action.

We now show that $\mathcal{V} /\left(i^{-1} \mathcal{I}_{Y}\right)^{p} \mathcal{V}$ is a quasi-coherent and flat $\mathcal{O}_{Y_{p}}$-module. Suppose first that $\left.\mathcal{V}_{Y}\right|_{U}$ is a free $\mathcal{O}_{U}$-module on $U$ so there exist sections $v_{j} \in$ $\Gamma\left(U, \mathcal{V}_{Y}\right), j \in J$, such that the map $\left.\mathcal{O}_{U}^{\oplus J} \rightarrow \mathcal{V}_{Y}\right|_{U},\left(f_{j}\right)_{j \in J} \mapsto \sum_{j \in J} f_{j} v_{j}$, is bijective. We define the map

$$
\psi:\left.\left(\left.\mathcal{O}_{Y_{p}}\right|_{U}\right)^{\oplus J} \rightarrow \prod_{s=0}^{p-1} \prod_{i \in I_{s, l}} \mathcal{V}_{Y}\right|_{U}
$$

by letting the $\boldsymbol{i}$-term of $\psi(f)$ for $\boldsymbol{i}=(i(1), \ldots, i(s)) \in I_{s, l}$ and $f=\left(f_{j}\right)_{j \in J}$ be

$$
\psi(f)_{i}=\left.\sum_{j \in J}\left(\left(\zeta_{i(1)}\right)_{X} \cdots\left(\zeta_{i(s)}\right)_{X} f_{j}\right)\right|_{U} \cdot v_{j} .
$$


Then $\psi$ is an isomorphism of $\left.\mathcal{O}_{Y_{p}}\right|_{U}$-modules and hence $\left.\left(\mathcal{V} /\left(i^{-1} \mathcal{I}_{Y}\right)^{p} \mathcal{V}\right)\right|_{U}$ is a free $\left.\mathcal{O}_{Y_{p}}\right|_{U}$-module.

For the general case, we write $V$ as a union of finite-dimensional $M$-submodules: $V=\bigcup_{\alpha} V^{\alpha}$. Then the $K$-equivariant quasi-coherent $\mathcal{O}_{Y}$-module $\mathcal{V}_{Y}^{\alpha}$ with fiber $V^{\alpha}$ is locally free. If we define the $\mathcal{O}_{Y_{p}}$-module structure on $\left.\prod_{s=0}^{p-1} \prod_{i \in I_{s, l}} \mathcal{V}_{Y}^{\alpha}\right|_{U}$ as in (5.4), then the preceding argument proves that it is a locally free $\left.\mathcal{O}_{Y_{p}}\right|_{U^{-}}$ module. Since $\mathcal{V}_{Y}$ is the union of $\mathcal{V}_{Y}^{\alpha}$, we see that $\left.\left(\mathcal{V} /\left(i^{-1} \mathcal{I}_{Y}\right)^{p} \mathcal{V}\right)\right|_{U}$ is isomorphic to the union of $\left.\prod_{s=0}^{p-1} \prod_{i \in I_{s, l}} \mathcal{V}_{Y}^{\alpha}\right|_{U}$ as an $\left.\mathcal{O}_{Y_{p}}\right|_{U}$-module. Hence $\mathcal{V} /\left(i^{-1} \mathcal{I}_{Y}\right)^{p} \mathcal{V}$ is a quasi-coherent and flat $\mathcal{O}_{Y_{p}}$-module.

We define a $K$-action on $\mathcal{V}$ by

$$
(k \cdot v)(f \otimes D)=k \cdot\left(v\left(\left(k^{-1} \cdot f\right) \otimes \operatorname{Ad}\left(i(k)^{-1}\right) D\right)\right)
$$

for $k \in K, v \in \mathcal{V}, f \in \mathcal{O}_{Y}$, and $D \in U(\mathfrak{g})$. This action descends to a $K$-action on $\mathcal{V} /\left(i^{-1} \mathcal{I}_{Y}\right)^{p} \mathcal{V}$ and makes it a $K$-equivariant $\mathcal{O}_{Y_{p}}$-module. From this definition, it immediately follows that the maps $\mathcal{V} /\left(i^{-1} \mathcal{I}_{Y}\right)^{p} \mathcal{V} \rightarrow \mathcal{V} /\left(i^{-1} \mathcal{I}_{Y}\right)^{p-1} \mathcal{V}$ and $i^{-1} \widetilde{\mathfrak{g}}_{X} \otimes$ $\mathcal{V} /\left(i^{-1} \mathcal{I}_{Y}\right)^{p} \mathcal{V} \rightarrow \mathcal{V} /\left(i^{-1} \mathcal{I}_{Y}\right)^{p-1} \mathcal{V}$ commute with $K$-actions for all $p>0$.

We have checked conditions (1), (2) and (3) of Definition 3.3. We can verify condition (4) by computing the $\mathfrak{k}$-action as

$$
\begin{aligned}
(\eta \cdot v)(f \otimes D) & =v(f \otimes D \eta) \\
& =-v(f \otimes[\eta, D])+v((1 \otimes \eta)(f \otimes D))-v\left(\left(\eta_{Y}(f)\right) \otimes D\right) \\
& =-v(f \otimes[\eta, D])+(1 \otimes \eta)(v(f \otimes D))-v\left(\left(\eta_{Y}(f)\right) \otimes D\right)
\end{aligned}
$$

for $\eta \in \mathfrak{k}, v \in \mathcal{V}, f \in \mathcal{O}_{Y}$, and $D \in U(\mathfrak{g})$.

For condition (5), we get an isomorphism of vector spaces $\iota: \mathcal{V} /\left(i^{-1} \mathcal{I}_{o}\right) \mathcal{V} \simeq V$ by taking the fiber of the isomorphism $\phi: \mathcal{V} /\left(i^{-1} \mathcal{I}_{Y}\right) \mathcal{V} \simeq \mathcal{V}_{Y}$ at $o$. The map $\iota$ is written as $\iota(v)=(v(1 \otimes 1))(e)$ for $v \in \mathcal{V}$. For $\xi \in \mathfrak{h}$, there exists a section $\xi^{\prime} \in i^{-1} \mathcal{H}$ near the base point $o$ such that $1 \otimes \xi-\xi^{\prime} \in i^{-1} \mathcal{I}_{o} \otimes \mathfrak{g}$, or equivalently, $\xi^{\prime}(e)=\xi$. Then

$$
\iota(\xi v)=((\xi v)(1 \otimes 1))(e)=(v(1 \otimes \xi))(e)=\left(v\left(\xi^{\prime}\right)\right)(e)=\xi(v(1 \otimes 1)(e))=\xi \iota(v) .
$$

Moreover, we have

$$
\iota(m v)=((m v)(1 \otimes 1))(e)=(m(v(1 \otimes 1)))(e)=m(v(1 \otimes 1)(e))=m \iota(v)
$$

for $m \in M$ and hence $\iota$ commutes with the $(\mathfrak{h}, M)$-actions.

Remark 5.3. The $i^{-1} \widetilde{\mathfrak{g}}_{X}$-module $\mathcal{V}$ constructed above in this section has the following universal property. If $\mathcal{V}^{\prime}$ is another $i^{-1} \widetilde{\mathfrak{g}}_{X}$-module associated with $V$, then there exists a canonical map $\mathcal{V}^{\prime} \rightarrow \mathcal{V}$ such that the induced map

$$
V \simeq \mathcal{V}^{\prime} /\left(i^{-1} \mathcal{I}_{o}\right) \mathcal{V}^{\prime} \rightarrow \mathcal{V} /\left(i^{-1} \mathcal{I}_{o}\right) \mathcal{V} \simeq V
$$


is the identity map. Moreover, it also induces an isomorphism

$$
\mathcal{V}^{\prime} /\left(i^{-1} \mathcal{I}_{Y}\right)^{p} \mathcal{V}^{\prime} \rightarrow \mathcal{V} /\left(i^{-1} \mathcal{I}_{Y}\right)^{p} \mathcal{V}
$$

for any $p \in \mathbb{N}$. Therefore, the tensor product $i^{-1} i_{+} \mathcal{L} \otimes_{i^{-1} \mathcal{O}_{X}} \mathcal{V}^{\prime}$ does not depend on the choice of $\mathcal{V}^{\prime}$ up to canonical isomorphism. We will give another description of the $i^{-1} \widetilde{\mathfrak{g}}_{X}$-module $i^{-1} i_{+} \mathcal{L} \otimes_{i^{-1} \mathcal{O}_{X}} \mathcal{V}$ in Proposition 6.1.

\section{$\S 6$. Twisted $\mathcal{D}$-modules}

Retain the notation of the previous sections. Let $V$ be an $(\mathfrak{h}, M)$-module and $\mathcal{V}$ an $i^{-1} \widetilde{\mathfrak{g}}_{X}$-module associated with $V$. Since $\mathcal{V} /\left(i^{-1} \mathcal{I}_{Y}\right) \mathcal{V}$ is a $K$-equivariant quasi-coherent $\mathcal{O}_{Y}$-module with typical fiber $V$, there is a canonical isomorphism $\mathcal{V} /\left(i^{-1} \mathcal{I}_{Y}\right) \mathcal{V} \simeq \mathcal{V}_{Y}$. We view $\mathcal{H}:=\operatorname{ker}\left(p: \mathcal{O}_{X} \otimes \mathfrak{g} \rightarrow \mathcal{T}_{X}\right)$ as a subsheaf of $U\left(\widetilde{\mathfrak{g}}_{X}\right)$. Since $\mathcal{H}\left(\mathcal{I}_{Y} \otimes U(\mathfrak{g})\right) \subset \mathcal{I}_{Y} \otimes U(\mathfrak{g})$, the $i^{-1} \mathcal{H}$-action on $\mathcal{V}$ induces one on $\mathcal{V} /\left(i^{-1} \mathcal{I}_{Y}\right) \mathcal{V}$. By regarding local sections of these equivariant modules as vector-valued regular functions, this action is written as

$$
(\xi v)(k)=\xi(i(k)) v(k)
$$

for $\xi \in i^{-1} \mathcal{H}, v \in \mathcal{V}$ and $k \in K$. Indeed, since the action map $i^{-1} \mathcal{H} \otimes \mathcal{V} /\left(i^{-1} \mathcal{I}_{Y}\right) \mathcal{V}$ $\rightarrow \mathcal{V} /\left(i^{-1} \mathcal{I}_{Y}\right) \mathcal{V}$ commutes with $K$-actions by Definition 3.3(3), it is enough to prove (6.1) for $k=e$. This follows from $\mathcal{H}\left(\mathcal{I}_{o} \otimes U(\mathfrak{g})\right) \subset \mathcal{I}_{o} \otimes U(\mathfrak{g})$ and Definition $3.3(5)$.

The $\mathcal{O}_{Y}$-modules $\mathcal{L}, \mathcal{V}_{Y}, \Omega_{Y}$, and $i^{*} \Omega_{X}^{\vee}$ are $K$-equivariant with typical fiber $\bigwedge^{\text {top }}(\mathfrak{k} / \mathfrak{l}), V, \Lambda^{\text {top }}(\mathfrak{k} / \mathfrak{m})^{*}$, and $\bigwedge^{\text {top }}(\mathfrak{g} / \mathfrak{h})$, respectively. Hence the tensor product $\mathcal{L} \otimes_{\mathcal{O}_{Y}} \mathcal{V}_{Y} \otimes_{\mathcal{O}_{Y}} \Omega_{Y} \otimes_{\mathcal{O}_{Y}} i^{*} \Omega_{X}^{\vee}$ is also $K$-equivariant and has typical fiber $\Lambda^{\text {top }}(\mathfrak{k} / \mathfrak{l}) \otimes$ $V \otimes \bigwedge^{\text {top }}(\mathfrak{k} / \mathfrak{m})^{*} \otimes \Lambda^{\text {top }}(\mathfrak{g} / \mathfrak{h})$. We define a right $i^{-1} \mathcal{H}$-module structure, a right $\mathfrak{k}$ module structure, and a right $\mathcal{O}_{Y}$-module structure on the sheaf $\mathcal{L} \otimes_{\mathcal{O}_{Y}} \mathcal{V}_{Y} \otimes_{\mathcal{O}_{Y}}$ $\Omega_{Y} \otimes_{\mathcal{O}_{Y}} i^{*} \Omega_{X}^{\vee}$ by

$$
\begin{aligned}
\left(\left(f \otimes v \otimes \omega \otimes \omega^{\prime}\right) \xi\right)(k)= & -f(k) \otimes(\xi(i(k)) v(k)) \otimes \omega(k) \otimes \omega^{\prime}(k) \\
& -f(k) \otimes v(k) \otimes \omega(k) \otimes \operatorname{ad}(\xi(i(k))) \omega^{\prime}(k), \\
\left(f \otimes v \otimes \omega \otimes \omega^{\prime}\right) \eta= & -(\eta f) \otimes v \otimes \omega \otimes \omega^{\prime}-f \otimes(\eta v) \otimes \omega \otimes \omega^{\prime} \\
& -f \otimes v \otimes(\eta \omega) \otimes \omega^{\prime}-f \otimes v \otimes \omega \otimes\left(\eta \omega^{\prime}\right), \\
\left(f \otimes v \otimes \omega \otimes \omega^{\prime}\right) f^{\prime}= & f^{\prime} f \otimes v \otimes \omega \otimes \omega^{\prime}
\end{aligned}
$$

for $f \in \mathcal{L}, \xi \in i^{-1} \mathcal{H}, \eta \in \mathfrak{k}, v \in \mathcal{V}_{Y}, \omega \in \Omega_{Y}, \omega^{\prime} \in i^{*} \Omega_{X}^{\vee}, f^{\prime} \in \mathcal{O}_{Y}$, and $k \in K$. These actions are compatible: if $f_{i} \in i^{-1} \mathcal{O}_{X}, \eta_{i} \in \mathfrak{k}$ and $\xi \in i^{-1} \mathcal{H}$ satisfy

$$
\sum_{i}\left(f_{i} \otimes \eta_{i}\right)-\xi \in i^{-1} \mathcal{I}_{Y} \otimes U(\mathfrak{g}),
$$


then we have

$$
\sum_{i}\left(\left.\left(f \otimes v \otimes \omega \otimes \omega^{\prime}\right) f_{i}\right|_{Y}\right) \eta_{i}=\left(f \otimes v \otimes \omega \otimes \omega^{\prime}\right) \xi
$$

Therefore, we can prove in the same way as in Section 5 that these actions define a right $\overline{\mathcal{A}}$-module structure on $\mathcal{L} \otimes_{\mathcal{O}_{Y}} \mathcal{V}_{Y} \otimes_{\mathcal{O}_{Y}} \Omega_{Y} \otimes_{\mathcal{O}_{Y}} i^{*} \Omega_{X}^{\vee}$.

By using this right $\overline{\mathcal{A}}$-module structure, we consider the sheaf

$$
\left(\mathcal{L} \otimes_{\mathcal{O}_{Y}} \mathcal{V}_{Y} \otimes_{\mathcal{O}_{Y}} \Omega_{Y} \otimes_{\mathcal{O}_{Y}} i^{*} \Omega_{X}^{\vee}\right) \otimes_{\overline{\mathcal{A}}}\left(\mathcal{O}_{Y} \otimes_{i^{-1} \mathcal{O}_{X}} i^{-1} U\left(\widetilde{\mathfrak{g}}_{X}\right)\right),
$$

which has a right $i^{-1} \widetilde{\mathfrak{g}}_{X}$-module structure. We view it as a left $i^{-1} \widetilde{\mathfrak{g}}_{X}$-module via the anti-isomorphism

$$
S: U\left(\widetilde{\mathfrak{g}}_{X}\right) \rightarrow U\left(\widetilde{\mathfrak{g}}_{X}\right), \quad f \otimes 1 \mapsto f \otimes 1, \quad 1 \otimes \xi \mapsto-1 \otimes \xi,
$$

for $f \in \mathcal{O}_{X}, \xi \in \mathfrak{g}$.

Proposition 6.1. Let $\mathcal{L}$ be as in Section 4. Let $\mathcal{V}$ be an $i^{-1} \widetilde{\mathfrak{g}}_{X}$-module associated with an $(\mathfrak{h}, M)$-module $V$. Then there exists a $K$-equivariant isomorphism of $i^{-1} \widetilde{\mathfrak{g}}_{X}$-modules

$i^{-1} i_{+} \mathcal{L} \otimes_{i^{-1} \mathcal{O}_{X}} \mathcal{V} \simeq\left(\mathcal{L} \otimes_{\mathcal{O}_{Y}} \mathcal{V}_{Y} \otimes_{\mathcal{O}_{Y}} \Omega_{Y} \otimes_{\mathcal{O}_{Y}} i^{*} \Omega_{X}^{\vee}\right) \otimes_{\overline{\mathcal{A}}}\left(\mathcal{O}_{Y} \otimes_{i^{-1} \mathcal{O}_{X}} i^{-1} U\left(\widetilde{\mathfrak{g}}_{X}\right)\right)$

Proof. Let $F_{p} i^{-1} i_{+} \mathcal{L}$ be the filtration of $i^{-1} i_{+} \mathcal{L}$ as in Section 4. Then $F_{0} i^{-1} i_{+} \mathcal{L}$ $\otimes_{i^{-1} \mathcal{O}_{X}} \mathcal{V}$ is regarded as a subsheaf of $i^{-1} i_{+} \mathcal{L} \otimes_{i^{-1} \mathcal{O}_{X}} \mathcal{V}$ (see Remark 4.2). We have

$$
\begin{aligned}
F_{0} i^{-1} i_{+} \mathcal{L} \otimes_{i^{-1} \mathcal{O}_{X}} \mathcal{V} & \simeq F_{0} i^{-1} i_{+} \mathcal{L} \otimes_{\mathcal{O}_{Y}} \mathcal{V} /\left(i^{-1} \mathcal{I}_{Y}\right) \mathcal{V} \\
& \simeq \mathcal{L} \otimes_{\mathcal{O}_{Y}} \Omega_{Y} \otimes_{\mathcal{O}_{Y}} i^{*} \Omega_{X}^{\vee} \otimes_{\mathcal{O}_{Y}} \mathcal{V} /\left(i^{-1} \mathcal{I}_{Y}\right) \mathcal{V}
\end{aligned}
$$

Therefore, we get an isomorphism of $K$-equivariant $\mathcal{O}_{Y}$-modules

$$
\begin{gathered}
\psi_{0}: \mathcal{L} \otimes_{\mathcal{O}_{Y}} \mathcal{V}_{Y} \otimes_{\mathcal{O}_{Y}} \Omega_{Y} \otimes_{\mathcal{O}_{Y}} i^{*} \Omega_{X}^{\vee} \stackrel{\sim}{\rightarrow} F_{0} i^{-1} i_{+} \mathcal{L} \otimes_{i^{-1} \mathcal{O}_{X}} \mathcal{V}, \\
f \otimes v \otimes \omega \otimes \omega^{\prime} \mapsto\left(f \otimes \omega \otimes \omega^{\prime}\right) \otimes v .
\end{gathered}
$$

Here $v \in \mathcal{V}_{Y}$ and we choose a section of $\mathcal{V}$ that is sent to $v \in \mathcal{V}_{Y} \simeq \mathcal{V} /\left(i^{-1} \mathcal{I}_{Y}\right) \mathcal{V}$ by the quotient map, which we denote by the same letter $v \in \mathcal{V}$. Write $\mathcal{V}_{Y}^{\prime}:=$ $\mathcal{L} \otimes \mathcal{O}_{Y} \mathcal{V}_{Y} \otimes \mathcal{O}_{Y} \Omega_{Y} \otimes \mathcal{O}_{Y} i^{*} \Omega_{X}^{\vee}$ for simplicity. The isomorphism (6.2) extends to the homomorphism of $i^{-1} \widetilde{\mathfrak{g}}_{X}$-modules

$$
\begin{aligned}
\psi: \mathcal{V}_{Y}^{\prime} \otimes_{\mathbb{C}}\left(\mathcal{O}_{Y} \otimes_{i^{-1} \mathcal{O}_{X}} i^{-1} U\left(\widetilde{\mathfrak{g}}_{X}\right)\right) & \rightarrow i^{-1} i_{+} \mathcal{L} \otimes_{i^{-1} \mathcal{O}_{X}} \mathcal{V}, \\
v \otimes(1 \otimes(f \otimes D)) & \mapsto S(f \otimes D) \cdot \psi_{0}(v)
\end{aligned}
$$

We can check that the map $\psi$ descends to

$$
\bar{\psi}: \mathcal{V}_{Y}^{\prime} \otimes_{\overline{\mathcal{A}}}\left(\mathcal{O}_{Y} \otimes_{i^{-1} \mathcal{O}_{X}} i^{-1} U\left(\widetilde{\mathfrak{g}}_{X}\right)\right) \rightarrow i^{-1} i_{+} \mathcal{L} \otimes_{i^{-1} \mathcal{O}_{X}} \mathcal{V}
$$


Let

$$
\pi: \mathcal{V}_{Y}^{\prime} \otimes_{\mathbb{C}}\left(\mathcal{O}_{Y} \otimes_{i^{-1} \mathcal{O}_{X}} i^{-1} U\left(\widetilde{\mathfrak{g}}_{X}\right)\right) \rightarrow \mathcal{V}_{Y}^{\prime} \otimes_{\overline{\mathcal{A}}}\left(\mathcal{O}_{Y} \otimes_{i^{-1} \mathcal{O}_{X}} i^{-1} U\left(\widetilde{\mathfrak{g}}_{X}\right)\right)
$$

be the quotient map and put

$$
\mathcal{V}_{p}:=\pi\left(\mathcal{V}_{Y}^{\prime} \otimes_{\mathbb{C}}\left(\mathcal{O}_{Y} \otimes_{\mathbb{C}} U_{p}(\mathfrak{g})\right)\right),
$$

where $\left\{U_{p}(\mathfrak{g})\right\}_{p \in \mathbb{N}}$ is the standard filtration of $U(\mathfrak{g})$. We have

$$
\bar{\psi}\left(\mathcal{V}_{p}\right)=\psi\left(\mathcal{V}_{Y}^{\prime} \otimes_{\mathbb{C}}\left(\mathcal{O}_{Y} \otimes_{\mathbb{C}} U_{p}(\mathfrak{g})\right) \subset F_{p} i^{-1} i_{+} \mathcal{L} \otimes_{i^{-1} \mathcal{O}_{X}} \mathcal{V} .\right.
$$

Let us take an open set $U \subset Y$ and elements $\zeta_{1}, \ldots, \zeta_{l} \in \mathfrak{g}$ as in the proof of Proposition 5.1 and use the same notation. Then by an argument similar to the proof of Proposition 5.1, we obtain a bijective map of sheaves

$$
\left.\left.\prod_{s=0}^{p} \prod_{i \in I_{s, l}} \mathcal{V}_{Y}^{\prime}\right|_{U} \simeq \mathcal{V}_{p}\right|_{U}, \quad\left(v_{\boldsymbol{i}}\right)_{\boldsymbol{i}} \mapsto \sum_{\boldsymbol{i}} \pi\left(v_{\boldsymbol{i}} \otimes\left(1 \otimes \zeta_{\boldsymbol{i}}\right)\right),
$$

and hence

$$
\left.\prod_{i \in I_{p, l}} \mathcal{V}_{Y}^{\prime}\right|_{U} \simeq \mathcal{V}_{p} /\left.\mathcal{V}_{p-1}\right|_{U}
$$

We also see that

$\left(F_{p} i^{-1} i_{+} \mathcal{L} \otimes_{i^{-1} \mathcal{O}_{X}} \mathcal{V}\right) /\left(F_{p-1} i^{-1} i_{+} \mathcal{L} \otimes_{i^{-1} \mathcal{O}_{X}} \mathcal{V}\right) \simeq\left(F_{p} i^{-1} i_{+} \mathcal{L} / F_{p-1} i^{-1} i_{+} \mathcal{L}\right) \otimes_{\mathcal{O}_{Y}} \mathcal{V}_{Y}$

and

$$
F_{p} i^{-1} i_{+} \mathcal{L} / F_{p-1} i^{-1} i_{+} \mathcal{L} \simeq \mathcal{L} \otimes_{\mathcal{O}_{Y}} \Omega_{Y} \otimes_{\mathcal{O}_{Y}} i^{*} \Omega_{X}^{\vee} \otimes_{\mathcal{O}_{Y}} i^{-1}\left(\left(\mathcal{I}_{Y}\right)^{p} /\left(\mathcal{I}_{Y}\right)^{p+1}\right)
$$

by [Osh11, Lemma 3.3]. Since $\zeta_{\boldsymbol{i}}$ for $\boldsymbol{i} \in I_{p, l}$ give a trivialization of $i^{-1}\left(\left(\mathcal{I}_{Y}\right)^{p} /\left(\mathcal{I}_{Y}\right)^{p+1}\right)$, we conclude that the map

$$
\mathcal{V}_{p} / \mathcal{V}_{p-1} \rightarrow\left(F_{p} i^{-1} i_{+} \mathcal{L} \otimes \mathcal{V}\right) /\left(F_{p-1} i^{-1} i_{+} \mathcal{L} \otimes \mathcal{V}\right)
$$

induced by $\bar{\psi}$ on the successive quotient is an isomorphism. Therefore the map $\bar{\psi}$ is also an isomorphism. We can also see that $\bar{\psi}$ commutes with the $K$-action. Hence the proposition follows.

Let $\lambda \in \mathfrak{h}^{*}$ be such that $\operatorname{Ad}^{*}(h) \lambda=\lambda$ for $h \in H$. For a section $\xi \in \mathcal{H}$, we define a function $f_{\xi, \lambda} \in \mathcal{O}_{X}$ as

$$
f_{\xi, \lambda}(g H)=\lambda(\xi(g)) .
$$

Let $\mathcal{I}_{\lambda}$ be the two-sided ideal of the sheaf $U\left(\widetilde{\mathfrak{g}}_{X}\right)=\mathcal{O}_{X} \otimes U(\mathfrak{g})$ generated by $\xi-\left(f_{\xi, \lambda} \otimes 1\right)$ for all $\xi \in \mathcal{H}$. We define the ring of twisted differential operators as

$$
\mathcal{D}_{X, \lambda}:=U\left(\widetilde{\mathfrak{g}}_{X}\right) / \mathcal{I}_{\lambda} .
$$


Let $\mu:=\left.\lambda\right|_{\mathfrak{m}}$ and define $\mathcal{D}_{Y, \mu}$ similarly. Then we can define the direct image of a left $\mathcal{D}_{Y, \mu}$-module $\mathcal{M}$ by

$$
i_{+} \mathcal{M}:=i_{*}\left(\left(\mathcal{M} \otimes_{\mathcal{O}_{Y}} \Omega_{Y}\right) \otimes_{\mathcal{D}_{Y,-\mu}} i^{*} \mathcal{D}_{X,-\lambda}\right) \otimes_{\mathcal{O}_{X}} \Omega_{X}^{\vee}
$$

Suppose that $V$ is an $(\mathfrak{h}, M)$-module and $\mathfrak{h}$ acts on $V$ by $\lambda \in \mathfrak{h}^{*}$. The $K$ equivariant $\mathcal{O}_{Y}$-module $\mathcal{L} \otimes_{\mathcal{O}_{Y}} \mathcal{V}_{Y}$ has a natural structure of left $\mathcal{D}_{Y, \mu}$-module. Therefore, we can define the direct image $i_{+}\left(\mathcal{L} \otimes \mathcal{O}_{Y} \mathcal{V}_{Y}\right)$ as a left $\mathcal{D}_{X, \lambda}$-module.

Proposition 6.2. Suppose that $V$ is an $(\mathfrak{h}, M)$-module and $\mathfrak{h}$ acts on $V$ by $\lambda \in \mathfrak{h}^{*}$ such that $\operatorname{Ad}^{*}(h) \lambda=\lambda$ for $h \in H$. Let $\mathcal{V}$ be an $i^{-1} \widetilde{\mathfrak{g}}_{X}$-module associated with $V$. Then we have a $K$-equivariant isomorphism of $i^{-1} \widetilde{\mathfrak{g}}_{X}$-modules

$$
i^{-1} i_{+} \mathcal{L} \otimes_{i^{-1} \mathcal{O}_{X}} \mathcal{V} \simeq i^{-1} i_{+}\left(\mathcal{L} \otimes_{\mathcal{O}_{Y}} \mathcal{V}_{Y}\right)
$$

Proof. We define a filtration $F_{p} i^{-1} i_{+}\left(\mathcal{L} \otimes \mathcal{O}_{Y} \mathcal{V}_{Y}\right)$ of $i^{-1} i_{+}\left(\mathcal{L} \otimes \mathcal{O}_{Y} \mathcal{V}_{Y}\right)$ in the same way as $F_{p} i^{-1} i_{+} \mathcal{L}$. Then

$$
F_{0} i^{-1} i_{+}\left(\mathcal{L} \otimes_{\mathcal{O}_{Y}} \mathcal{V}_{Y}\right) \simeq \mathcal{L} \otimes_{\mathcal{O}_{Y}} \mathcal{V}_{Y} \otimes_{\mathcal{O}_{Y}} \Omega_{Y} \otimes_{\mathcal{O}_{Y}} i^{*} \Omega_{X}^{\vee}
$$

By using the same argument as in Proposition 6.1, we define a map of $i^{-1} \widetilde{\mathfrak{g}}_{X^{-}}$ modules

$$
\mathcal{V}_{Y}^{\prime} \otimes_{\mathbb{C}}\left(\mathcal{O}_{Y} \otimes_{i^{-1} \mathcal{O}_{X}} i^{-1} U\left(\widetilde{\mathfrak{g}}_{X}\right)\right) \rightarrow i^{-1} i_{+}\left(\mathcal{L} \otimes_{\mathcal{O}_{Y}} \mathcal{V}_{Y}\right)
$$

and we see that it induces an isomorphism

$$
\mathcal{V}_{Y}^{\prime} \otimes_{\overline{\mathcal{A}}}\left(\mathcal{O}_{Y} \otimes_{i^{-1} \mathcal{O}_{X}} i^{-1} U\left(\widetilde{\mathfrak{g}}_{X}\right)\right) \simeq i^{-1} i_{+}\left(\mathcal{L} \otimes_{\mathcal{O}_{Y}} \mathcal{V}_{Y}\right)
$$

Hence

$$
i^{-1} i_{+} \mathcal{L} \otimes_{i^{-1} \mathcal{O}_{X}} \mathcal{V} \simeq i^{-1} i_{+}\left(\mathcal{L} \otimes_{\mathcal{O}_{Y}} \mathcal{V}_{Y}\right)
$$

by Proposition 6.1.

Recall that $\mathcal{L}$ is the $K$-equivariant invertible sheaf on $Y=K / M$ with typical fiber $\bigwedge^{\text {top }}(\mathfrak{k} / \mathfrak{l})$. We view a one-dimensional vector space $\bigwedge^{\text {top }}(\mathfrak{k} / \mathfrak{l})^{*}$ as an $(\mathfrak{h}, M)$ module in the following way: $\mathfrak{h}$ acts as zero; the Levi component $L$ of $M$ acts as the coadjoint action $\bigwedge \mathrm{Ad}^{*}$; the unipotent radical $U$ of $M$ acts trivially. Let $\mathcal{L}^{\prime}$ be an $i^{-1} \widetilde{\mathfrak{g}}_{X}$-module associated with $\bigwedge^{\text {top }}(\mathfrak{k} / \mathfrak{l})^{*}$. Then $\mathcal{L}^{\prime} /\left(i^{-1} \mathcal{I}_{Y}\right) \mathcal{L}^{\prime}$ is isomorphic to the dual of $\mathcal{L}$. Therefore, by Proposition 6.2 we have

$$
i^{-1} i_{+} \mathcal{L} \otimes_{i^{-1} \mathcal{O}_{X}} \mathcal{V} \otimes_{i^{-1} \mathcal{O}_{X}} \mathcal{L}^{\prime} \simeq i^{-1} i_{+} \mathcal{V}_{Y}
$$

Example 3.6 shows that the $i^{-1} \widetilde{\mathfrak{g}}_{X}$-module $\mathcal{V} \otimes_{i^{-1}} \mathcal{O}_{X} \mathcal{L}^{\prime}$ is associated with $V \otimes$ $\bigwedge^{\text {top }}(\mathfrak{k} / \mathfrak{l})^{*}$. 
Theorem 6.3. In Setting 3.2, assume that $K$ is reductive. Suppose that $V$ is an $(\mathfrak{h}, M)$-module and $\mathfrak{h}$ acts on $V$ by $\lambda \in \mathfrak{h}^{*}$ such that $\operatorname{Ad}^{*}(h) \lambda=\lambda$ for $h \in H$. Let $M=L \ltimes U$ be a Levi decomposition. Then

$$
\begin{aligned}
\mathrm{H}^{s}\left(Y, i^{-1} i_{+} \mathcal{V}_{Y}\right) & \simeq\left(P_{\mathfrak{h}, L}^{\mathfrak{g}, K}\right)_{u-s}\left(V \otimes \bigwedge^{\mathrm{top}}(\mathfrak{k} / \mathfrak{l})^{*} \otimes \bigwedge^{\mathrm{top}}(\mathfrak{g} / \mathfrak{h})\right) \\
& \simeq\left(I_{\mathfrak{g}, L}^{\mathfrak{g}, K}\right)^{y+s} P_{\mathfrak{h}, L}^{\mathfrak{g}, L}\left(V \otimes \bigwedge^{\mathrm{top}}(\mathfrak{g} / \mathfrak{h})\right)
\end{aligned}
$$

for $s \in \mathbb{N}, u=\operatorname{dim} U$, and $y=\operatorname{dim} Y$.

Proof. The first isomorphism follows from Theorem 4.1 and the argument above. Since the functor $P_{\mathfrak{h}, L}^{\mathfrak{g}, L}$ is exact, $\left(P_{\mathfrak{h}, L}^{\mathfrak{g}, K}\right)_{u-s} \simeq\left(P_{\mathfrak{g}, L}^{\mathfrak{g}, K}\right)_{u-s} \circ P_{\mathfrak{h}, L}^{\mathfrak{g}, L}$. Hence the duality ([KV95, Theorem 3.5])

$$
\left(P_{\mathfrak{g}, L}^{\mathfrak{g}, K}\right)_{\operatorname{dim}(K / L)-s}\left(\cdot \otimes \bigwedge^{\text {top }}(\mathfrak{k} / \mathfrak{l})^{*}\right) \simeq\left(I_{\mathfrak{g}, L}^{\mathfrak{g}, K}\right)^{s}(\cdot)
$$

and $\operatorname{dim} K / L=\operatorname{dim} U+\operatorname{dim} Y$ give the second isomorphism.

By Theorem 6.3 we obtain the convergence of the spectral sequence

$$
\begin{aligned}
\mathrm{H}^{s}\left(X, R^{t} i_{+} \mathcal{V}_{Y}\right) & \Rightarrow\left(P_{\mathfrak{h}, L}^{\mathfrak{g}, K}\right)_{u-s-t}\left(V \otimes \bigwedge^{\text {top }}(\mathfrak{k} / \mathfrak{l})^{*} \otimes \bigwedge^{\text {top }}(\mathfrak{g} / \mathfrak{h})\right) \\
& \simeq\left(I_{\mathfrak{g}, L}^{\mathfrak{g}, K}\right)^{y+s+t} P_{\mathfrak{h}, L}^{\mathfrak{g}, L}\left(V \otimes \bigwedge^{\text {top }}(\mathfrak{g} / \mathfrak{h})\right) .
\end{aligned}
$$

Here $R^{t} i_{+}$is the higher direct image functor for a twisted left $\mathcal{D}$-module.

We will now see that this spectral sequence implies results of [HMSW87] and [Kit10].

Example 6.4. Let $G_{\mathbb{R}}$ be a connected real semisimple Lie group with a maximal compact subgroup $K_{\mathbb{R}}$ and the complexified Lie algebra $\mathfrak{g}$. Let $K$ be the complexification of $K_{\mathbb{R}}$ and $G$ the inner automorphism group of $\mathfrak{g}$. There is a canonical homomorphism $i: K \rightarrow G$, which has finite kernel. Suppose that $H$ is a Borel subgroup of $G$. Let us apply Setting 3.2. Then $X=G / H$ is the full flag variety of $\mathfrak{g}$. Since $L$ is abelian and $K$ is connected, $L$ acts trivially on $\bigwedge^{\text {top }}(\mathfrak{k} / \mathfrak{l})^{*}$. Moreover in this case it is known that $Y$ is affinely embedded in $X$. Therefore, $R^{t} i_{+} \simeq 0$ for $t>0$ and the spectral sequence (6.3) collapses. We thus get (1.1) and hence the duality theorem (Theorem 1.1).

Example 6.5. Let $G_{\mathbb{R}}$ be a connected real semisimple Lie group with a maximal compact subgroup $K_{\mathbb{R}}$. We define $K, G$, and $i: K \rightarrow G$ as in the previous example. Suppose that $H$ is a parabolic subgroup of $G$ and apply Setting 3.2. Then $X=$ $G / H$ is a partial flag variety of $\mathfrak{g}$. In this case $Y$ is not necessarily affinely embedded in $X$. Let $\widetilde{X}$ be the full flag variety of $\mathfrak{g}$ and let $p: \widetilde{X} \rightarrow X$ be the natural surjective map. Then we have an isomorphism $\mathrm{H}^{s}\left(\widetilde{X}, p^{*} \mathcal{M}\right) \simeq \mathrm{H}^{s}(X, \mathcal{M})$ for any 
$\mathcal{O}_{X}$-module $\mathcal{M}$. Hence $(6.3)$ becomes

$$
\mathrm{H}^{s}\left(\widetilde{X}, p^{*} R^{t} i_{+} \mathcal{V}_{Y}\right) \Rightarrow\left(I_{\mathfrak{g}, L}^{\mathfrak{g}, K}\right)^{y+s+t} P_{\mathfrak{h}, L}^{\mathfrak{g}, L}\left(V \otimes \bigwedge^{\text {top }}(\mathfrak{g} / \mathfrak{h})\right),
$$

which is [Kit10, Theorem 25 (6.6)].

Let $V$ be any $(\mathfrak{h}, M)$-module and $\mathcal{V}$ an $i^{-1} \widetilde{\mathfrak{g}}_{X}$-module associated with $V$. Since $i^{-1} i_{+} \mathcal{L} \otimes_{i^{-1} \mathcal{O}_{X}} \mathcal{L}^{\prime} \simeq i^{-1} i_{+} \mathcal{O}_{Y}$, we have

$$
i^{-1} i_{+} \mathcal{L} \otimes_{i^{-1} \mathcal{O}_{X}} \mathcal{L}^{\prime} \otimes_{i^{-1} \mathcal{O}_{X}} \mathcal{V} \simeq i^{-1} i_{+} \mathcal{O}_{Y} \otimes_{i^{-1} \mathcal{O}_{X}} \mathcal{V}
$$

We can thus rewrite Theorem 4.1 as

Theorem 6.6. In Setting 3.2, assume that $K$ is reductive. Let $M=L \ltimes U$ be a Levi decomposition. Suppose that $V$ is an $(\mathfrak{h}, M)$-module and $\mathcal{V}$ is an $i^{-1} \widetilde{\mathfrak{g}}_{X}$-module associated with $V$ (Definition 3.3). Then

$$
\begin{aligned}
\mathrm{H}^{s}\left(Y, i^{-1} i_{+} \mathcal{O}_{Y} \otimes_{i^{-1}} \mathcal{O}_{X} \mathcal{V}\right) & \simeq\left(P_{\mathfrak{h}, L}^{\mathfrak{g}, K}\right)_{u-s}\left(V \otimes \bigwedge^{\mathrm{top}}(\mathfrak{k} / \mathfrak{l})^{*} \otimes \bigwedge^{\text {top }}(\mathfrak{g} / \mathfrak{h})\right) \\
& \simeq\left(I_{\mathfrak{g}, L}^{\mathfrak{g}, K}\right)^{y+s} P_{\mathfrak{h}, L}^{\mathfrak{g}, L}\left(V \otimes \bigwedge^{\mathrm{top}}(\mathfrak{g} / \mathfrak{h})\right)
\end{aligned}
$$

for $s \in \mathbb{N}, u=\operatorname{dim} U, y=\operatorname{dim} Y$.

\section{Acknowledgements}

The author was supported by Grant-in-Aid for JSPS Fellows (10J00710).

\section{References}

[BB81] A. Beı̌linson and J. Bernstein, Localisation de $g$-modules, C. R. Acad. Sci. Paris Sér. I Math. 292 (1981), 15-18. Zbl 0476.14019 MR 0610137

[BB93] A A proof of Jantzen conjectures, in I. M. Gel'fand Seminar, Adv. Soviet Math. 16, Amer. Math. Soc., Providence, RI, 1993, 1-50. Zbl 0790.22007 MR 1237825

[BL95] J. Bernstein and V. Lunts, Localization for derived categories of $(\mathfrak{g}, K)$-modules, J. Amer. Math. Soc. 8 (1995), 819-856. Zbl 0852.22015 MR 1317229

[Bie90] F. V. Bien, D-modules and spherical representations, Math. Notes 39, Princeton Univ. Press, Princeton, NJ, 1990. Zbl 0723.22014 MR 1082342

[BL95] J. Bernstein and V. Lunts, Localization for derived categories of ( $\mathfrak{g}, K)$-modules, J. Amer. Math. Soc. 8 (1995), 819-856. Zbl 0852.22015 MR 1317229

[Cha93] J.-T. Chang, Remarks on localization and standard modules: the duality theorem on a generalized flag variety, Proc. Amer. Math. Soc. 117 (1993), 585-591. Zbl 0833.22020 MR 1145942

[HMSW87] H. Hecht, D. Miličić, W. Schmid, and J. A. Wolf, Localization and standard modules for real semisimple Lie groups. I. The duality theorem, Invent. Math. 90 (1987), 297332. Zbl 0699.22022 MR 0910203

[Kit10] S. N. Kitchen, Localization of cohomologically induced modules to partial flag varieties, Ph.D. Thesis, The University of Utah, 2010. MR 2736734 
[KV95] A. W. Knapp and D. A. Vogan, Jr., Cohomological induction and unitary representations, Princeton Math. Ser. 45, Princeton Univ. Press, Princeton, NJ, 1995. Zbl 0863.22011 MR 1330919

[MP98] D. Miličić and P. Pandžić, Equivariant derived categories, Zuckerman functors and localization, in Geometry and representation theory of real and p-adic groups (Córdoba, 1995), Progr. Math. 158, Birkhäuser Boston, Boston, MA, 1998, 209-242. Zbl 0907.22019 MR 1486143

[Osh11] Y. Oshima, On the restriction of Zuckerman's derived functor modules $A_{\mathfrak{q}}(\lambda)$ to reductive subgroups, arXiv:1107.2833.

[Sch91] W. Schmid, Construction and classification of irreducible Harish-Chandra modules, in Harmonic analysis on reductive groups (Brunswick, ME, 1989), Progr. Math. 101, Birkhäuser Boston, Boston, MA, 1991, 235-275. Zbl 0751.22003 MR 1168487 